\title{
A GENERAL ASYMPTOTIC DYNAMIC MODEL FOR LIPSCHITZIAN ELASTIC CURVED RODS
}

\author{
ROSTISLAV VODÁK
}

Received 31 June 2005 and in revised form 15 November 2005

We study the asymptotic behaviour of solutions to the linear evolution problem for clamped curved rods with the small thickness $\epsilon$ under minimal regularity assumptions on the geometry. In addition, nonconstant density of the curved rods is considered.

\section{Introduction}

The main task of this paper is to relax regularity assumptions on a shape of elastic curved rods in a general asymptotic dynamic model and to derive this asymptotic model from a linear evolution equation of three dimensional elasticity by asymptotic technique.

We use the asymptotic approach presented by Aganovic and Tutek [17] for straight rods, which was modified by Jurak and Tambača $[8,9]$ (see also Trabucho and Viaño [16]) and by Ignat, Sprekels, and Tiba [7] for curved rods generated by a function $\Phi \in$ $C^{k}([0, l])^{3}, k \geq 3$, and $k \geq 2$, respectively. The approach from $[8,9]$ was applied to a dynamic model of curved rods in [13]. Following an idea from Blouza and Le Dret [3], a new formulation of the equations of elasticity which required $\Phi \in C^{2}([0, l])^{3}$ was found in Tiba and Vodák [14]. In addition, the general asymptotic model obtained in [14] was well-defined for a Jordan unit speed curve with Lipschitzian parameterization which led to a special construction of its approximations with smooth Jordan unit speed curves. The whole construction was valid for $\Phi_{i}^{\prime}>0$ a.e. on $(0, l)$ for some $i, i=1,2,3$. This restrictive condition was excluded by the modification of the construction in [15]. In this paper, we want to extend the theory presented in $[14,15]$ on the dynamic model for the curved rods. Among other papers concerning with the dynamic models for thin curved structures we mention here Raoult [10], Xiao [18], and Sprekels and Tiba [12]. Further we recommend the reader Álvarez-Dios and Viaño [1], Bermúdez and Viaño [2].

Finally, we give a brief outline of the paper. In Section 2, we introduce the basic notations and notions that will be further needed. Section 3 contains auxiliary propositions, which are used throughout the paper. Section 4 is devoted to a weak formulation of the linear elasticity equation and its transformation. Section 5 deals with basic estimates. Section 6 gives us a basic overview about behaviour of the displacements if $\epsilon \rightarrow 0$ and about qualitative properties of their limit state. In Section 7, the passage to the limit $\epsilon \rightarrow 0$ is performed and the main existence and uniqueness result is proved. 
The main result of this paper can be summarized in the following theorem.

Theorem 1.1. Let a function $\Phi \in W^{1, \infty}(0, l)^{3}$ be a parametrization of a unit speed curve. Let, further, $\mathbf{F} \in L^{2}\left(0, T ; L^{2}(\Omega)^{3}\right), \mathbf{G} \in W^{1,1}\left(0, T ; L^{2}\left(0, l ; L^{2}(\partial S)^{3}\right)\right)$ and $\check{\mathbf{F}}_{\mathbf{F}+\mathbf{G}}$ be defined as in Lemma 7.4. Then, there is a unique pair $\langle\mathbf{U}, \phi\rangle \in L^{\infty}\left(0, T ; \mathscr{V}_{0}^{\mathbf{t}, \mathbf{n}, \mathbf{b}}(0, l)\right)$ such that $\partial_{t} \mathbf{U} \in$ $L^{\infty}\left(0, T ; L^{2}(0, l)^{3}\right) \cap C\left([0, T] ;\left[\mathscr{V}_{0}^{\mathbf{t}, \mathbf{n}, \mathbf{b}}(0, l)\right]^{\prime}\right)$, which generates a unique solution to the problem (7.41)-(7.42). Moreover, the constant extension to $\Omega=(0, l) \times S$ of $\langle\mathbf{U}, \phi\rangle$ may be approximated by solutions $\mathbf{U}_{\epsilon} \in L^{\infty}\left(0, T ; V(\Omega)^{3}\right) \cap W^{1, \infty}\left(0, T ; L^{2}(\Omega)^{3}\right)$ of the problem (4.13)(4.14) as follows:

$$
\begin{gathered}
\mathbf{U}=\lim _{\epsilon \rightarrow 0} \mathbf{U}_{\epsilon} * \text {-weakly in } L^{\infty}\left(0, T ; V(\Omega)^{3}\right), \\
\partial_{t} \mathbf{U}=\lim _{\epsilon \rightarrow 0} \partial_{t} \mathbf{U}_{\epsilon} * \text {-weakly in } L^{\infty}\left(0, T ; L^{2}(\Omega)^{3}\right), \\
\phi=\lim _{\epsilon \rightarrow 0} \frac{1}{2 \epsilon}\left(\left(\partial_{2} \mathbf{U}_{\epsilon}, \mathbf{b}_{\epsilon}\right)-\left(\partial_{3} \mathbf{U}_{\epsilon}, \mathbf{n}_{\epsilon}\right)\right) * \text {-weakly in } L^{\infty}\left(0, T ; L^{2}(\Omega)\right) .
\end{gathered}
$$

\section{Preliminaries}

We denote by $\mathbb{R}^{3}$ the usual three-dimensional Euclidean space with scalar product $(\cdot, \cdot)$ and norm $|\cdot|$. Let $S \subset \mathbb{R}^{2}$ be a bounded simply connected domain of class $C^{1}$ satisfying the "symmetry" condition

$$
\int_{S} x_{2} d x_{2} d x_{3}=\int_{S} x_{3} d x_{2} d x_{3}=\int_{S} x_{2} x_{3} d x_{2} d x_{3}=0
$$

We denote by $\Omega=(0, l) \times S, \Omega_{\epsilon}=(0, l) \times \epsilon S$ open cylinders in $\mathbb{R}^{3}$, where $l>0$ and $\epsilon>0$ "small," are given.

Let a function $\Phi:[0, l] \rightarrow \mathbb{R}^{3}, \boldsymbol{\Phi} \in W^{1, \infty}(0, l)^{3}$, be a parametrization of a Jordan unit speed curve $\mathscr{C}$ in $\mathbb{R}^{3}$ and let $\mathbf{t}, \mathbf{n}, \mathbf{b}$ denote its tangent, normal and binormal vectors. Let $\Phi_{\epsilon}:[0, l] \rightarrow \mathbb{R}^{3}$ be a smoothing of $\boldsymbol{\Phi}$ such that it remains a Jordan unit speed curve (i.e., $\left.\left|\Phi_{\epsilon}^{\prime}\left(y_{1}\right)\right|=1, \forall y_{1} \in[0, l]\right)$ and $\mathbf{t}_{\epsilon}, \mathbf{n}_{\epsilon}, \mathbf{b}_{\epsilon}$ be the associated local frame. Alternative ways, how to construct local frames under low regularity assumptions, may be found in [12]. The whole construction of the local frame associated with the function $\boldsymbol{\Phi}$ and its smoothing can be found in $[14,15]$. Here we mention only the needed properties of the approximation: $\left\{\boldsymbol{\Phi}_{\epsilon}\right\}_{\epsilon \in(0,1)},\left\{\mathbf{t}_{\epsilon}\right\}_{\epsilon \in(0,1)},\left\{\mathbf{n}_{\epsilon}\right\}_{\epsilon \in(0,1)},\left\{\mathbf{b}_{\epsilon}\right\}_{\epsilon \in(0,1)} \subset C^{\infty}([0, l])^{3}$,

$$
\begin{gathered}
\mathbf{t}_{\epsilon} \longrightarrow \mathbf{t}, \quad \mathbf{n}_{\epsilon} \longrightarrow \mathbf{n}, \quad \mathbf{b}_{\epsilon} \longrightarrow \mathbf{b} \quad \text { in measure in }(0, l), \\
\boldsymbol{\Phi}_{\epsilon}(\cdot)=\int_{0} \mathbf{t}_{\epsilon}(z) d z+\boldsymbol{\Phi}(0) \longrightarrow \boldsymbol{\Phi}(\cdot) \quad \text { in } C([0, l])^{3}
\end{gathered}
$$

for $\epsilon \rightarrow 0$,

$$
\left\|\mathbf{t}_{\epsilon}^{\prime}\right\|_{\infty}+\left\|\mathbf{n}_{\epsilon}^{\prime}\right\|_{\infty}+\left\|\mathbf{b}_{\epsilon}^{\prime}\right\|_{\infty} \leq \frac{C}{\epsilon^{r}}, \quad\left\|\mathbf{t}_{\epsilon}^{\prime \prime}\right\|_{\infty}+\left\|\mathbf{n}_{\epsilon}^{\prime \prime}\right\|_{\infty}+\left\|\mathbf{b}_{\epsilon}^{\prime \prime}\right\|_{\infty} \leq \frac{C}{\epsilon^{2 r}}, \quad r \in\left(0, \frac{1}{3}\right)
$$

The whole construction can be found in $[14,15]$. 
The orthogonality properties $\left(\mathbf{t}_{\epsilon}, \mathbf{t}_{\epsilon}^{\prime}\right)=0,\left(\mathbf{n}_{\epsilon}, \mathbf{n}_{\epsilon}^{\prime}\right)=0,\left(\mathbf{b}_{\epsilon}, \mathbf{b}_{\epsilon}^{\prime}\right)=0$ lead to so called "laws of motion" of the local frame

$$
\begin{aligned}
\mathbf{t}_{\epsilon}^{\prime} & =\alpha_{\epsilon} \mathbf{b}_{\epsilon}+\beta_{\epsilon} \mathbf{n}_{\epsilon}, \\
\mathbf{n}_{\epsilon}^{\prime} & =-\beta_{\epsilon} \mathbf{t}_{\epsilon}-\gamma_{\epsilon} \mathbf{b}_{\epsilon}, \\
\mathbf{b}_{\epsilon}^{\prime} & =-\alpha_{\epsilon} \mathbf{t}_{\epsilon}+\gamma_{\epsilon} \mathbf{n}_{\epsilon} .
\end{aligned}
$$

From (2.4), it follows that

$$
\left\|\alpha_{\epsilon}\right\|_{\infty}+\left\|\beta_{\epsilon}\right\|_{\infty}+\left\|\gamma_{\epsilon}\right\|_{\infty} \leq \frac{C}{\epsilon^{r}}, \quad\left\|\alpha_{\epsilon}^{\prime}\right\|_{\infty}+\left\|\beta_{\epsilon}^{\prime}\right\|_{\infty}+\left\|\gamma_{\epsilon}^{\prime}\right\|_{\infty} \leq \frac{C}{\epsilon^{2 r}}, \quad r \in\left(0, \frac{1}{3}\right) .
$$

We adopt the usual notation for the standard Sobolev and Lebesgue spaces, that is, $H^{1}(\Omega)$, $H_{0}^{1}(\Omega)$ and $L^{p}(\Omega), p \in[1, \infty]$ for the spaces and $\|\cdot\|_{1,2},\|\cdot\|_{p}$ for their norms. We will use the same notation of the norms also for vector or tensor functions in the case that all their components belong to the above mentioned Sobolev or Lebesgue spaces. $H^{-1}(\Omega)$ and $[X]^{\prime}$ stand for the dual space to $H_{0}^{1}(\Omega)$ or $X$, respectively. The notation $C^{m}(\bar{\Omega})$, with $m \in \mathbb{N}_{0}$, means the usual spaces of continuous functions whose derivatives up to the order $m$ are continuous in $\bar{\Omega}$, and we denote by $C_{0}^{\infty}(\Omega)$ the space of all functions which have derivatives of any order on $\Omega$ and whose supports are compact subsets of $\Omega$. The symbols $L^{p}(I ; X), p \in[1, \infty]$ and $C(I ; X)$, where $X$ is a Banach space and $I$ is a bounded interval, stand for the Bochner spaces endowed with the norms $\|v\|_{L^{p}(I ; X)}$ and $\|v\|_{C(\bar{I} ; X)}$, respectively. We say that $v \in C\left([0, T] ; L_{\text {weak }}^{2}(\Omega)\right)$ if the function $\int_{\Omega} v(t) w d x$ is continuous on $[0, T]$ for an arbitrary function $w \in L^{2}(\Omega)$.

Except for the standard definition of the weak convergence in $X$ or $L^{p}(I ; X), p \in(1, \infty)$, and $*$-weak convergence in $L^{\infty}\left(I,[X]^{\prime}\right)$, we say without danger of confusion that $v_{n} \rightarrow v$ in $L^{2}\left(0, l ; H^{-1}(S)\right)$, if

$$
\int_{0}^{l} H^{-1}(S)\left\langle v_{n}\left(x_{1}\right)-v\left(x_{1}\right), \psi\left(x_{1}\right)\right\rangle_{H_{0}^{1}(S)} d x_{1} \longrightarrow 0 \quad \text { for any } \psi \in L^{2}\left(0, l ; H_{0}^{1}(S)\right),
$$

where $[X]^{\prime}\langle\cdot, \cdot\rangle_{X}$ denotes the dual pairing of $[X]^{\prime}$ and $X$, and $v_{n} \stackrel{*}{\rightarrow} v$ in $L^{\infty}\left(I, L^{2}(\Omega)\right)$ if

$$
\int_{I} \int_{\Omega}\left(v_{n}-v\right) \psi d x d z \longrightarrow 0
$$

for any $\psi \in L^{1}\left(I, L^{2}(\Omega)\right)$. Further, we introduce the space

$$
\begin{aligned}
\mathscr{V}_{0}^{\mathbf{t}, \mathbf{n}, \mathbf{b}}(0, l)=\left\{\lfloor\mathbf{V}, \psi\rfloor \in H_{0}^{1}(0, l)^{3} \times L^{2}(0, l):\left(\mathbf{V}^{\prime}, \mathbf{t}\right)=0,\right. \\
\\
\left.\mathbf{V}_{*}=-\psi \mathbf{t}+\left(\mathbf{V}^{\prime}, \mathbf{b}\right) \mathbf{n}-\left(\mathbf{V}^{\prime}, \mathbf{n}\right) \mathbf{b} \in H_{0}^{1}(0, l)^{3}\right\}
\end{aligned}
$$

We refer the reader to [14] for the proof that $\mathscr{V}_{0}^{\mathbf{t}, \mathbf{n}, \mathbf{b}}(0, l)$ is a nontrivial Hilbert space endowed with the norm

$$
\|[\mathbf{V}, \psi\rfloor\|^{2}=\|\mathbf{V}\|_{1,2}^{2}+\|\psi\|_{2}^{2}+\left\|\mathbf{V}_{*}\right\|_{1,2}^{2} .
$$


Let $v \in L_{\mathrm{loc}}^{1}(0, T)$ and $\varphi \in C_{0}^{\infty}(0, T)$. Then we denote $\bar{v}^{\varphi}=\int_{0}^{T} v(t) \varphi(t) d t$. Now, we introduce the mappings $\mathbf{R}_{\epsilon}$ and $\overline{\mathbf{P}}_{\epsilon}$,

$$
\begin{gathered}
\mathbf{R}_{\epsilon}: \Omega \longrightarrow \Omega_{\epsilon}, \quad \mathbf{R}_{\epsilon}\left(x_{1}, x_{2}, x_{3}\right)=\left(x_{1}, \epsilon x_{2}, \epsilon x_{3}\right), \\
\overline{\mathbf{P}}_{\epsilon}: \Omega_{\epsilon} \longrightarrow \mathbb{R}^{3}, \quad \overline{\mathbf{P}}_{\epsilon}(y)=\boldsymbol{\Phi}_{\epsilon}\left(y_{1}\right)+y_{2} \mathbf{n}_{\epsilon}\left(y_{1}\right)+y_{3} \mathbf{b}_{\epsilon}\left(y_{1}\right),
\end{gathered}
$$

$\left(y_{1}, y_{2}, y_{3}\right) \in \Omega_{\epsilon}=(0, l) \times \epsilon S$, where the second one gives the parametrization of the curved $\operatorname{rod} \widetilde{\Omega}_{\epsilon}=\overline{\mathbf{P}}_{\epsilon}\left(\Omega_{\epsilon}\right)$. Furthermore,

$$
\bar{d}_{\epsilon}(y)=\operatorname{det}\left(\bar{\nabla} \overline{\mathbf{P}}_{\epsilon}(y)\right)=1-\beta_{\epsilon}\left(y_{1}\right) y_{2}-\alpha_{\epsilon}\left(y_{1}\right) y_{3} \quad \forall y \in \bar{\Omega}_{\epsilon} .
$$

We can suppose that $\bar{d}_{\epsilon}(y)>0$ for all $y \in \bar{\Omega}_{\epsilon}$ and for $\epsilon$ "small" (see (2.6) and the definition of $\Omega_{\epsilon}$ ). Then $\overline{\mathbf{P}}_{\epsilon}: \Omega_{\epsilon} \rightarrow \widetilde{\Omega}_{\epsilon}$ is a $C^{1}$-diffeomorphism according to Ciarlet [4, Theorem 3.1-1]. In the sequel, we will write $\tilde{\partial}_{i}=\partial / \partial \tilde{y}_{i}$, where $\tilde{y}=\left(\tilde{y}_{1}, \tilde{y}_{2}, \tilde{y}_{3}\right) \in \widetilde{\Omega}_{\epsilon}, \bar{\partial}_{i}=\partial / \partial y_{i}$, for $y=\left(y_{1}, y_{2}, y_{3}\right) \in \Omega_{\epsilon}, \partial_{i}=\partial / \partial x_{i}$, where $x=\left(x_{1}, x_{2}, x_{3}\right) \in \Omega, \partial_{t}=\partial / \partial t$ and $\partial_{t t}=\partial^{2} / \partial t^{2}$. Thus, in $(2.13), \bar{\nabla}=\left(\bar{\partial}_{1}, \bar{\partial}_{2}, \bar{\partial}_{3}\right)$. In the case that a function $v$ depends only on $t$ or $x_{1}$ (or $\left.y_{1}\right)$, we denote its first (second) derivation by $\dot{v}(\ddot{v})$ and $v^{\prime}\left(v^{\prime \prime}\right)$, respectively. Sometimes it is more convenient to use the notation $(d / d t) v$ instead of $\dot{v}$.

The definition of the domain $\widetilde{\Omega}_{\epsilon}$ enables us to introduce the following spaces:

$$
\begin{gathered}
V\left(\widetilde{\Omega}_{\epsilon}\right)=\left\{\tilde{V} \in H^{1}\left(\widetilde{\Omega}_{\epsilon}\right):\left.\tilde{V}\right|_{\overline{\mathbf{P}}_{\epsilon}(\{0\} \times \epsilon S)}=\left.\tilde{V}\right|_{\overline{\mathbf{P}}_{\epsilon}(\{l\} \times \epsilon S)}=0\right\}, \\
V(\Omega)=\left\{V \in H^{1}(\Omega):\left.V\right|_{(\{0\} \times S)}=\left.V\right|_{(\{l\} \times S)}=0\right\} .
\end{gathered}
$$

In an analogous way as above, we denote by $\widetilde{V}$ a function defined on $\widetilde{\Omega}_{\epsilon}, \bar{V}$ a function defined on $\Omega_{\epsilon}$, and $V$ a function defined on $\Omega$.

The covariant and the contravariant basis at the point $\overline{\mathbf{P}}_{\epsilon}(y), y \in \Omega_{\epsilon}$, of the curved rod are defined by $\overline{\mathbf{g}}_{i, \epsilon}(y)=\bar{\partial}_{i} \overline{\mathbf{P}}_{\epsilon}(y)$ and $\left(\overline{\mathbf{g}}_{i, \epsilon}, \overline{\mathbf{g}}^{j, \epsilon}\right)=\delta^{i j}$, and (using (2.5)) these vectors are given by

$$
\begin{gathered}
\overline{\mathbf{g}}_{1, \epsilon}(y)=\left(1-y_{2} \beta_{\epsilon}\left(y_{1}\right)-y_{3} \alpha_{\epsilon}\left(y_{1}\right)\right) \mathbf{t}_{\epsilon}\left(y_{1}\right)+y_{3} \gamma_{\epsilon}\left(y_{1}\right) \mathbf{n}_{\epsilon}\left(y_{1}\right)-y_{2} \gamma_{\epsilon}\left(y_{1}\right) \mathbf{b}_{\epsilon}\left(y_{1}\right), \\
\overline{\mathbf{g}}_{2, \epsilon}(y)=\mathbf{n}_{\epsilon}\left(y_{1}\right), \quad \overline{\mathbf{g}}_{3, \epsilon}(y)=\mathbf{b}_{\epsilon}\left(y_{1}\right), \\
\overline{\mathbf{g}}^{1, \epsilon}(y)=\frac{\mathbf{t}_{\epsilon}\left(y_{1}\right)}{\bar{d}_{\epsilon}(y)}, \quad \overline{\mathbf{g}}^{2, \epsilon}(y)=-\frac{y_{3} \gamma_{\epsilon}\left(y_{1}\right) \mathbf{t}_{\epsilon}\left(y_{1}\right)}{\bar{d}_{\epsilon}(y)}+\mathbf{n}_{\epsilon}\left(y_{1}\right) \\
\overline{\mathbf{g}}^{3, \epsilon}(y)=\frac{y_{2} \gamma_{\epsilon}\left(y_{1}\right) \mathbf{t}_{\epsilon}\left(y_{1}\right)}{\bar{d}_{\epsilon}(y)}+\mathbf{b}_{\epsilon}\left(y_{1}\right) .
\end{gathered}
$$

Further, we define the covariant and contravariant metric tensors $\left(\bar{g}_{i j, \epsilon}\right)_{i, j=1}^{3},\left(\bar{g}^{i j, \epsilon}\right)_{i, j=1}^{3}$, where

$$
\bar{g}_{i j, \epsilon}=\left(\overline{\mathbf{g}}_{i, \epsilon}, \overline{\mathbf{g}}_{j, \epsilon}\right), \quad \bar{g}^{i j, \epsilon}=\left(\overline{\mathbf{g}}^{i, \epsilon}, \overline{\mathbf{g}}^{j, \epsilon}\right) .
$$


After substitution $y=\mathbf{R}_{\epsilon}(x)$, we adopt the notation

$$
\begin{gathered}
g^{i j, \epsilon}(x)=\bar{g}^{i j, \epsilon}\left(\mathbf{R}_{\epsilon}(x)\right), \quad g_{i j, \epsilon}(x)=\bar{g}_{i j, \epsilon}\left(\mathbf{R}_{\epsilon}(x)\right), \quad \mathbf{g}_{i, \epsilon}(x)=\overline{\mathbf{g}}_{i, \epsilon}\left(\mathbf{R}_{\epsilon}(x)\right), \\
\mathbf{g}^{j, \epsilon}(x)=\overline{\mathbf{g}}^{j, \epsilon}\left(\mathbf{R}_{\epsilon}(x)\right), \quad d_{\epsilon}(x)=\bar{d}_{\epsilon}\left(\mathbf{R}_{\epsilon}(x)\right),
\end{gathered}
$$

where $x \in \Omega$. Analogously as above, we can find the contravariant tensor $o^{i j, \epsilon}$ for the mapping $\overline{\mathbf{P}}_{\epsilon} \circ \mathbf{R}_{\epsilon}$ having the form

$$
\left(o^{i j, \epsilon}\right)_{i, j=1}^{3}=\left(\begin{array}{ccc}
\frac{1}{d_{\epsilon}^{2}} & -\frac{x_{3} \gamma_{\epsilon}}{d_{\epsilon}^{2}} & \frac{x_{2} \gamma_{\epsilon}}{d_{\epsilon}^{2}} \\
-\frac{x_{3} \gamma_{\epsilon}}{d_{\epsilon}^{2}} & \frac{1}{\epsilon^{2}}+\frac{x_{3}^{2} \gamma_{\epsilon}^{2}}{d_{\epsilon}^{2}} & -\frac{x_{2} x_{3} \gamma_{\epsilon}^{2}}{d_{\epsilon}^{2}} \\
\frac{x_{2} \gamma_{\epsilon}}{d_{\epsilon}^{2}} & -\frac{x_{2} x_{3} \gamma_{\epsilon}^{2}}{d_{\epsilon}^{2}} & \frac{1}{\epsilon^{2}}+\frac{x_{2}^{2} \gamma_{\epsilon}^{2}}{d_{\epsilon}^{2}}
\end{array}\right) .
$$

By " $X$ " we will denote the Cartesian product of two spaces and by $\lfloor\cdot, \cdot\rfloor$ any ordered pair. In the text, the symbol $|A|$ will also denote the Lebesgue measure of some measurable set $A$, without danger of confusion. The summation convention with respect to repeated indices will be also used, if not otherwise explicitly stated. We use for constants the symbols $C$ or $C_{i}$, for $i \in \mathbb{N}_{0}=\{0,1,2, \ldots\}$.

\section{Auxiliary propositions}

Proposition 3.1 [14]. Let $\mathbf{t}_{\epsilon}, \mathbf{n}_{\epsilon}, \mathbf{b}_{\epsilon}$ satisfy (2.2)-(2.6) and let the space $\mathscr{V}_{0}^{\mathbf{t}_{\epsilon}, \mathbf{n}_{\epsilon}, \mathbf{b}_{\epsilon}}(0, l)$ be defined by (2.9) using the functions $\mathbf{t}_{\epsilon}, \mathbf{n}_{\epsilon}, \mathbf{b}_{\epsilon}$ instead of $\mathbf{t}, \mathbf{n}, \mathbf{b}$. Let, further, $\lfloor\mathbf{V}, \psi\rfloor \in \mathcal{V}_{0}^{\mathbf{t}, \mathbf{n}, \mathbf{b}}(0, l)$ be an arbitrary but fixed couple. Then there exist the couples

$$
\left\lfloor\mathbf{V}_{\epsilon}, \psi_{\epsilon}\right\rfloor \in \mathcal{V}_{0}^{\mathbf{t}_{\epsilon}, \mathbf{n}_{\epsilon}, \mathbf{b}_{\epsilon}}(0, l)
$$

generating the functions $\mathbf{V}_{*, \epsilon}$ such that $\left\{\mathbf{V}_{\epsilon}\right\}_{\epsilon \in(0,1)},\left\{\mathbf{V}_{*, \epsilon}\right\}_{\epsilon \in(0,1)} \subset C_{0}^{\infty}(0, l)^{3},\left\{\psi_{\epsilon}\right\}_{\epsilon \in(0,1)} \subset$ $C_{0}^{\infty}(0, l)$,

$$
\begin{gathered}
\mathbf{V}_{\epsilon} \longrightarrow \mathbf{V}, \quad \mathbf{V}_{*, \epsilon} \longrightarrow \mathbf{V}_{*} \quad \text { in } H_{0}^{1}(0, l)^{3} \\
\psi_{\epsilon} \longrightarrow \psi \quad \text { in measure on }(0, l),
\end{gathered}
$$

for $\epsilon \rightarrow 0$, and

$$
\left\|\mathbf{V}_{\epsilon}^{\prime \prime}\right\|_{2}+\left\|\psi_{\epsilon}^{\prime}\right\|_{2} \leq \frac{C}{\epsilon^{r}}, \quad r \in\left(0, \frac{1}{3}\right)
$$

Proposition 3.2 [14]. Let $\lambda \geq 0, \mu>0$ and

$$
A_{\epsilon}^{i j k l}=\lambda g^{i j, \epsilon} g^{k l, \epsilon}+\mu\left(g^{i k, \epsilon} g^{j l, \epsilon}+g^{i l, \epsilon} g^{j k, \epsilon}\right) .
$$

Then there exists a constant $C_{3}>0$ such that the estimate

$$
\sum_{i, j=1}^{3}\left|t_{i j}\right|^{2} \leq C_{3} A_{\epsilon}^{i j k l}(x) t_{k l} t_{i j}
$$


holds for all $x \in \bar{\Omega}$, all $\epsilon \in[0,1]$ and all symmetric matrices $\left(t_{i j}\right)_{i, j=1}^{3}$, with the constant $C_{3}$ being independent of $\epsilon$ and $x$.

Proposition 3.3 [14]. There exists a constant $C_{4}>0$ independent of $\epsilon$ such that

$$
\|\mathbf{V}\|_{1,2} \leq \frac{C_{4}}{\epsilon}\left\|\omega^{\epsilon}(\mathbf{V})\right\|_{2}, \quad \forall \mathbf{V} \in V(\Omega)^{3}, \forall \epsilon \in(0,1) .
$$

Proposition 3.4 [9]. Let $\left\{v_{n}\right\}_{n=1}^{\infty} \subset L^{2}\left(0, l ; L^{2}(S)\right),\left\{\partial_{1} v_{n}\right\}_{n=1}^{\infty} \subset L^{2}\left(0, l ; H^{-1}(S)\right)$ be such that $\left.v_{n}\right|_{x_{1}=0}=\left.v_{n}\right|_{x_{1}=l}=0$, for all $n \in \mathbb{N}$, in the sense of the space $C\left([0, l] ; H^{-1}(S)\right)$. Assume, in addition, that these sequences satisfy

$$
\partial_{1} v_{n}-\xi, \quad \partial_{j} v_{n} \longrightarrow 0, \quad \text { in } L^{2}\left(0, l ; H^{-1}(S)\right), j=2,3,
$$

where $\xi \in L^{2}\left(0, l ; H^{-1}(S)\right)$. Then $\xi \in L^{2}(0, l)$, and there exists a unique function $v \in H_{0}^{1}(0, l)$ such that $v^{\prime}=\xi$ and

$$
\begin{gathered}
v_{n} \longrightarrow v \quad \text { in } L^{2}\left(0, l ; L^{2}(S)\right), \\
v_{n} \longrightarrow v \quad \text { in } C\left([0, l] ; H^{-1}(S)\right) .
\end{gathered}
$$

If the convergences in (3.8) are strong then the convergence (3.9) is also strong.

Proposition 3.5 [14]. We have

$$
\begin{gathered}
d_{\epsilon} \longrightarrow 1 \quad \text { in } C(\bar{\Omega}), \\
\epsilon d_{\epsilon}(x) \sqrt{v_{i}(x) o^{i j, \epsilon}(x) v_{j}(x)} \longrightarrow 1 \quad \text { in } C(\overline{(0, l) \times \partial S}),
\end{gathered}
$$

for $\epsilon \rightarrow 0$, where $v_{i}, i=1,2,3$, are components of a unit outward normal to $(0, l) \times \partial S$. Thus, there exist constants $C_{j}, j=0,1,2$, such that $0<C_{0} \leq d_{\epsilon}(x) \leq C_{1}$ for all $x \in \bar{\Omega}$, and $0 \leq$ $d_{\epsilon}(x) \epsilon \sqrt{v_{i}(x) o^{i j, \epsilon}(x) v_{j}(x)} \leq C_{2}$ for all $x \in \overline{(0, l) \times \partial S}$ and $\epsilon \in(0,1)$.

\section{Weak formulation of an evolution equation for the curved rods and its transformation}

We consider $\widetilde{\Omega}_{\epsilon}$ defined by mapping $\overline{\mathbf{P}}_{\epsilon} \circ \mathbf{R}_{\epsilon}$ (see (2.11)-(2.12)) for $\epsilon \in(0,1)$ arbitrary but fixed as a three-dimensional homogeneous and isotropic elastic body with the Lamé constants $\lambda \geq 0, \mu>0$ and with mass density $\tilde{\rho}_{\epsilon}$. Let $\widetilde{\mathbf{F}}_{\epsilon}$ be the body force and $\widetilde{\mathbf{G}}_{\epsilon}$ the surface traction acting on the curved $\operatorname{rod} \widetilde{\Omega}_{\epsilon}$ such that $\widetilde{\mathbf{F}}_{\epsilon} \in L^{2}\left(0, T ; L^{2}\left(\widetilde{\Omega}_{\epsilon}\right)^{3}\right)$ and $\widetilde{\mathbf{G}}_{\epsilon} \in$ $W^{1,1}\left(0, T ; L^{2}\left(\left(\overline{\mathbf{P}}_{\epsilon} \circ \mathbf{R}_{\epsilon}\right)((0, l) \times \partial S)\right)^{3}\right)$, for $\epsilon \in(0,1)$. Let $\widetilde{\Omega}_{\epsilon}$ be clamped on both bases $\overline{\mathbf{P}}_{\epsilon}(\{0\} \times \epsilon S)$ and $\overline{\mathbf{P}}_{\epsilon}(\{l\} \times \epsilon S)$. The equilibrium displacement $\widetilde{\mathbf{U}}_{\epsilon}$ is a (weak) solution of the equation

$$
\begin{array}{r}
\int_{0}^{T} \int_{\tilde{\Omega}_{\epsilon}}\left[-\left(\tilde{\rho}_{\epsilon} \partial_{t} \widetilde{\mathbf{U}}_{\epsilon}(t), \partial_{t} \tilde{\mathbf{V}}(t)\right)+\widetilde{A}^{i j k l} e_{k l}\left(\widetilde{\mathbf{U}}_{\epsilon}(t)\right) e_{i j}(\tilde{\mathbf{V}}(t))\right] d \tilde{y} d t \\
=\int_{0}^{T} \int_{\tilde{\Omega}_{\epsilon}}\left(\widetilde{\mathbf{F}}_{\epsilon}(t), \tilde{\mathbf{V}}(t)\right) d \tilde{y} d t+\int_{0}^{T} \int_{\widetilde{S}_{\epsilon}}\left(\widetilde{\mathbf{G}}_{\epsilon}(t), \tilde{\mathbf{V}}(t)\right) d \widetilde{S}_{\epsilon} d \tilde{y}_{1} d t
\end{array}
$$


for all $\tilde{\mathbf{V}} \in C_{0}^{\infty}\left(0, T ; V\left(\widetilde{\Omega}_{\epsilon}\right)^{3}\right)$, where $\widetilde{S}_{\epsilon}=\left(\overline{\mathbf{P}}_{\epsilon} \circ \mathbf{R}_{\epsilon}\right)((0, l) \times \partial S), \tilde{A}^{i j k l}=\lambda \delta^{i j} \delta^{k l}+\mu\left(\delta^{i k} \delta^{j l}+\right.$ $\left.\delta^{i l} \delta^{j k}\right)$ and $\left(e_{i j}(\tilde{\mathbf{V}})\right)_{i, j=1}^{3}$ stands for the symmetric part of the gradient of the function $\tilde{\mathbf{V}}$. The solution $\tilde{\mathbf{U}}_{\epsilon}$ satisfies the initial state

$$
\left.\widetilde{\mathbf{U}}_{\epsilon}\right|_{t=0}=\widetilde{\mathbf{Q}}_{0, \epsilon},\left.\quad \partial_{t} \widetilde{\mathbf{U}}_{\epsilon}\right|_{t=0}=\widetilde{\mathbf{Q}}_{1, \epsilon}
$$

Using the fact that the functions $\mathbf{t}_{\epsilon}, \mathbf{n}_{\epsilon}, \mathbf{b}_{\epsilon} \in C^{\infty}([0, l])^{3}$ together with (2.11), (2.12), it is easy to see that the mapping $\overline{\mathbf{P}}_{\epsilon} \circ \mathbf{R}_{\epsilon}$ is the parametrization of the smooth threedimensional curved rod.

We transform now (4.1). Denoting $\mathbf{U}_{\epsilon}=\tilde{\mathbf{U}}_{\epsilon}\left(\overline{\mathbf{P}}_{\epsilon} \circ \mathbf{R}_{\epsilon}\right), \rho_{\epsilon}=\tilde{\rho}_{\epsilon}\left(\overline{\mathbf{P}}_{\epsilon} \circ \mathbf{R}_{\epsilon}\right)$ and $\mathbf{V}_{\epsilon}=$ $\tilde{\mathbf{V}}\left(\overline{\mathbf{P}}_{\epsilon} \circ \mathbf{R}_{\epsilon}\right)$, we get

$$
\int_{0}^{T} \int_{\tilde{\Omega}_{\epsilon}}\left(\tilde{\rho}_{\epsilon} \partial_{t} \tilde{\mathbf{U}}_{\epsilon}(t), \partial_{t} \tilde{\mathbf{V}}(t)\right) d \tilde{y} d t=\int_{0}^{T} \int_{\Omega}\left(\rho_{\epsilon} \partial_{t} \mathbf{U}_{\epsilon}(t), \partial_{t} \mathbf{V}_{\epsilon}(t)\right) \epsilon^{2} d_{\epsilon} d x d t
$$

For the transformation of other terms we refer the reader to [14]. It is easy to see that if $\tilde{\mathbf{V}} \in C_{0}^{\infty}\left(0, T ; V\left(\widetilde{\Omega}_{\epsilon}\right)^{3}\right)$, then $\mathbf{V}_{\epsilon} \in C_{0}^{\infty}\left(0, T ; V(\Omega)^{3}\right)$. Denoting $\mathbf{Q}_{0, \epsilon}=\widetilde{\mathbf{Q}}_{0, \epsilon}\left(\overline{\mathbf{P}}_{\epsilon} \circ \mathbf{R}_{\epsilon}\right), \mathbf{Q}_{1, \epsilon}=$ $\widetilde{\mathbf{Q}}_{1, \epsilon}\left(\overline{\mathbf{P}}_{\epsilon} \circ \mathbf{R}_{\epsilon}\right), \mathbf{F}_{\epsilon}=\widetilde{\mathbf{F}}_{\epsilon}\left(\overline{\mathbf{P}}_{\epsilon} \circ \mathbf{R}_{\epsilon}\right)$ and $\mathbf{G}_{\epsilon}=\widetilde{\mathbf{G}}_{\epsilon}\left(\overline{\mathbf{P}}_{\epsilon} \circ \mathbf{R}_{\epsilon}\right)$, we can rewrite the model (4.1)(4.2) using (4.3) and the transformation from [14] as

$$
\begin{aligned}
\int_{0}^{T} \int_{\Omega} & -\left(\rho_{\epsilon} \partial_{t} \mathbf{U}_{\epsilon}(t), \partial_{t} \mathbf{V}(t)\right) d_{\epsilon}+A_{\epsilon}^{i j k l} \omega_{k l}^{\epsilon}\left(\mathbf{U}_{\epsilon}(t)\right) \omega_{i j}^{\epsilon}\left(\mathbf{V}_{\epsilon}(t)\right) d_{\epsilon} d x d t \\
= & \int_{0}^{T} \int_{\Omega}\left(\mathbf{F}_{\epsilon}(t), \mathbf{V}(t)\right) d_{\epsilon} d x d t+\int_{0}^{T} \int_{0}^{l} \int_{\partial S}\left(\mathbf{G}_{\epsilon}(t), \mathbf{V}(t)\right) d_{\epsilon} \sqrt{v_{i} o^{i j, \epsilon} v_{j}} d S d x_{1} d t
\end{aligned}
$$

for all $\mathbf{V} \in C_{0}^{\infty}\left(0, T ; V(\Omega)^{3}\right)$, where the solution $\mathbf{U}_{\epsilon}$ satisfies the initial state

$$
\left.\mathbf{U}_{\epsilon}\right|_{t=0}=\mathbf{Q}_{0, \epsilon},\left.\quad \partial_{t} \mathbf{U}_{\epsilon}\right|_{t=0}=\mathbf{Q}_{1, \epsilon},
$$

$\nu_{i}, i=1,2,3$, are the components of the unit outward normal to $(0, l) \times \partial S,\left(o^{i j, \epsilon}\right)_{i, j=1}^{3}$ was introduced in (2.21), and where the symmetric tensor $\omega^{\epsilon}(\mathbf{V})$ has the form

$$
\omega^{\epsilon}(\mathbf{V})=\frac{1}{\epsilon} \theta^{\epsilon}(\mathbf{V})+\kappa^{\epsilon}(\mathbf{V})
$$

The individual nonzero components of the symmetric tensors $\theta^{\epsilon}$ and $\kappa^{\epsilon}$ are defined by

$$
\begin{gathered}
\theta_{12}^{\epsilon}(\mathbf{V})=\frac{1}{2}\left(\partial_{2} \mathbf{V}, \mathbf{g}_{1, \epsilon}\right), \quad \theta_{22}^{\epsilon}(\mathbf{V})=\left(\partial_{2} \mathbf{V}, \mathbf{n}_{\epsilon}\right), \quad \theta_{33}^{\epsilon}(\mathbf{V})=\left(\partial_{3} \mathbf{V}, \mathbf{b}_{\epsilon}\right), \\
\theta_{13}^{\epsilon}(\mathbf{V})=\frac{1}{2}\left(\partial_{3} \mathbf{V}, \mathbf{g}_{1, \epsilon}\right), \quad \theta_{23}^{\epsilon}(\mathbf{V})=\frac{1}{2}\left(\left(\partial_{2} \mathbf{V}, \mathbf{b}_{\epsilon}\right)+\left(\partial_{3} \mathbf{V}, \mathbf{n}_{\epsilon}\right)\right), \\
\kappa_{11}^{\epsilon}(\mathbf{V})=\left(\partial_{1} \mathbf{V}, \mathbf{g}_{1, \epsilon}\right), \quad \kappa_{12}^{\epsilon}(\mathbf{V})=\frac{1}{2}\left(\partial_{1} \mathbf{V}, \mathbf{n}_{\epsilon}\right), \quad \kappa_{13}^{\epsilon}(\mathbf{V})=\frac{1}{2}\left(\partial_{1} \mathbf{V}, \mathbf{b}_{\epsilon}\right) .
\end{gathered}
$$

Assumptions. The following assumptions will be needed throughout the paper:

(1) $\rho_{\epsilon}=\epsilon^{2} \rho$, where $\rho \in L^{\infty}(\Omega)$ and

$$
0<C_{5} \leq \rho \leq C_{6} \quad \text { a.e. in } \Omega \text {; }
$$


432 An asymptotic dynamic model for curved rods

(2) $\mathbf{F}_{\epsilon}=\epsilon^{2} \mathbf{F}, \mathbf{F} \in L^{2}\left(0, T ; L^{2}(\Omega)^{3}\right), \mathbf{G}_{\epsilon}=\epsilon^{3} \mathbf{G}, \mathbf{G} \in W^{1,1}\left(0, T ; L^{2}\left(0, l ; L^{2}(\partial S)^{3}\right)\right)$;

(3) $\left\{\mathbf{Q}_{0, \epsilon}\right\}_{\epsilon \in(0,1)} \subset V(\Omega)^{3},\left\{\mathbf{Q}_{1, \epsilon}\right\}_{\epsilon \in(0,1)} \subset L^{2}(\Omega)^{3}$,

$$
\frac{1}{\epsilon}\left\|\omega^{\epsilon}\left(\mathbf{Q}_{0, \epsilon}\right)\right\|_{2} \leq C, \quad \forall \epsilon \in(0,1),
$$

where the constant $C$ is independent of $\epsilon$, and

$$
\mathbf{Q}_{0, \epsilon} \rightarrow \mathbf{Q}_{0} \quad \text { in } V(\Omega)^{3}, \quad \mathbf{Q}_{1, \epsilon} \rightarrow \mathbf{Q}_{1} \quad \text { in } L^{2}(\Omega)^{3}
$$

for $\epsilon \rightarrow 0$, where $\mathbf{Q}_{0} \in H_{0}^{1}(0, l)^{3}$ and $\mathbf{Q}_{1} \in L^{2}(0, l)^{3}$, that is, the functions $\mathbf{Q}_{0}, \mathbf{Q}_{1}$ are the constant functions in the second and third variable.

The reason for the choice of these scalings can be found in the inequalities (3.7) and (5.1). We are not able to guarantee boundedness of the functions $\mathbf{U}_{\epsilon}$ in appropriate spaces without the scalings, which means that the curved rod can be broken when the diameter converges to zero.

After substitution of the above assumptions to (4.4)-(4.5), we get

$$
\begin{aligned}
& \int_{0}^{T} \int_{\Omega} {\left[-\left(\rho \partial_{t} \mathbf{U}_{\epsilon}(t), \partial_{t} \mathbf{V}(t)\right) d_{\epsilon}+A_{\epsilon}^{i j k l} \frac{1}{\epsilon} \omega_{k l}^{\epsilon}\left(\mathbf{U}_{\epsilon}(t)\right) \frac{1}{\epsilon} \omega_{i j}^{\epsilon}\left(\mathbf{V}_{\epsilon}(t)\right) d_{\epsilon}\right] d x d t } \\
&=\int_{0}^{T} \int_{\Omega}(\mathbf{F}(t), \mathbf{V}(t)) d_{\epsilon} d x d t+\int_{0}^{T} \int_{0}^{l} \int_{\partial S}(\mathbf{G}(t), \mathbf{V}(t)) d_{\epsilon} \epsilon \sqrt{v_{i} o^{i j, \epsilon} v_{j}} d S d x_{1} d t
\end{aligned}
$$

for all $\mathbf{V} \in C_{0}^{\infty}\left(0, T ; V(\Omega)^{3}\right)$, and

$$
\left.\mathbf{U}_{\epsilon}\right|_{t=0}=\mathbf{Q}_{0, \epsilon},\left.\quad \partial_{t} \mathbf{U}_{\epsilon}\right|_{t=0}=\mathbf{Q}_{1, \epsilon}
$$

\section{Basic estimates of a solution to (4.13)-(4.14)}

Proposition 5.1. Under the assumptions of Section 4, there exists a unique weak solution $\mathbf{U}_{\epsilon}$ to the problem (4.13)-(4.14) such that $\mathbf{U}_{\epsilon} \in L^{\infty}\left(0, T ; V(\Omega)^{3}\right), \partial_{t} \mathbf{U}_{\epsilon} \in L^{\infty}\left(0, T ; L^{2}(\Omega)^{3}\right)$, $\rho \partial_{t t} \mathbf{U}_{\epsilon} \in L^{2}\left(0, T ;\left[V(\Omega)^{3}\right]^{\prime}\right)$, where the initial conditions in (4.14) are fulfilled in the sense of the space $C\left([0, T] ; L^{2}(\Omega)^{3}\right)$ or $C\left([0, T] ; L_{\text {weak }}^{2}(\Omega)^{3}\right)$, respectively. In addition, this solution satisfies for all $\epsilon \in(0,1)$ the estimates

$$
\begin{aligned}
& \left\|\partial_{t} \mathbf{U}_{\epsilon}\right\|_{L^{\infty}\left(0, T ; L^{2}(\Omega)^{3}\right)}^{2}+\left\|\frac{1}{\epsilon} \omega\left(\mathbf{U}_{\epsilon}\right)\right\|_{L^{\infty}\left(0, T ; L^{2}(\Omega)^{9}\right)}^{2} \\
& \quad \leq C\left(\left\|\mathbf{Q}_{1, \epsilon}\right\|_{2}^{2}+\left\|\frac{1}{\epsilon} \omega\left(\mathbf{Q}_{0, \epsilon}\right)\right\|_{2}^{2}+\|\mathbf{F}\|_{L^{2}\left(0, T ; L^{2}(\Omega)^{3}\right)}^{2}+\|\mathbf{G}\|_{W^{1,1}\left(0, T ; L^{2}\left(0, l ; L^{2}(\partial S)^{3}\right)\right)}^{2}\right) \\
& \left\|\rho \partial_{t t} \mathbf{U}_{\epsilon}\right\|_{L^{2}\left(0, T ;\left[V(\Omega)^{3}\right]^{\prime}\right)} \\
& \quad \leq C\left(\|\mathbf{F}\|_{L^{2}\left(0, T ; L^{2}(\Omega)^{3}\right)}+\|\mathbf{G}\|_{L^{2}\left(0, T ; L^{2}\left(0, l ; L^{2}(\partial S)^{3}\right)\right)}+\frac{1}{\epsilon^{2}}\left\|\omega^{\epsilon}\left(\mathbf{U}_{\epsilon}\right)\right\|_{L^{2}\left(0, T ; L^{2}(\Omega)^{9}\right)}\right)
\end{aligned}
$$

where the constant $C$ is independent of $\epsilon$. 
Before we start to prove Proposition 5.1, we construct a finite dimensional approximation of the weak solution to our problem using analogous arguments as in $[5,6]$, and we prove an auxiliary lemma, which enable us to prove Proposition 5.1.

Let $\epsilon \in(0,1)$ be arbitrary but fixed. Since the space $V(\Omega)$ is a separable Hilbert space with the scalar product $((V, W))_{\rho d_{\epsilon}, \Omega}=\int_{\Omega} \rho V W d_{\epsilon} d x+\int_{\Omega} \rho(\nabla V, \nabla W) d_{\epsilon} d x$, we can select smooth functions $W_{k}, k=1,2, \ldots$, such that

$$
\left\{W_{k}\right\}_{k=1}^{\infty} \text { is a basis of } V(\Omega) \text { and an orthonormal basis of } L^{2}(\Omega)
$$

in the sense of the scalar product $(V, W)_{\rho d_{\epsilon}, \Omega}=\int_{\Omega} \rho V W d_{\epsilon} d x$. The proof that the above mentioned scalar products are well-defined follows from (3.11) and (4.10).

Now, we fix a positive integer $m$, and we write

$$
\begin{gathered}
U_{\epsilon, j}^{m}(x, t)=\sum_{k=1}^{m} d_{\epsilon, k, j}^{m}(t) W_{k}(x), \quad Q_{0, \epsilon, j}^{m}(x)=\sum_{k=1}^{m} d_{\epsilon, k, j}^{m}(0) W_{k}(x), \\
Q_{1, \epsilon, j}^{m}(x)=\sum_{k=1}^{m} \dot{d}_{\epsilon, k, j}^{m}(0) W_{k}(x),
\end{gathered}
$$

where $j=1,2,3,(x, t) \in \Omega \times(0, T)$ and $\mathbf{U}_{\epsilon}^{m}=\left(U_{\epsilon, 1}^{m}, U_{\epsilon, 2}^{m}, U_{\epsilon, 3}^{m}\right)$. Using the vectors $\mathbf{W}_{k}^{1}=$ $\left(W_{k}, 0,0\right), \mathbf{W}_{k}^{2}=\left(0, W_{k}, 0\right), \mathbf{W}_{k}^{3}=\left(0,0, W_{k}\right)$, we will study the system of equations

$$
\begin{gathered}
\int_{\Omega} \rho \partial_{t t} U_{\epsilon, \hat{i}}^{m}(t) W_{k} d_{\epsilon} d x+\int_{\Omega} A_{\epsilon}^{i j k l} \frac{1}{\epsilon} \omega_{k l}^{\epsilon}\left(\mathbf{U}_{\epsilon}^{m}(t)\right) \frac{1}{\epsilon} \omega_{i j}^{\epsilon}\left(\mathbf{W}_{k}^{\hat{i}}\right) d_{\epsilon} d x \\
=\int_{\Omega} F_{\hat{i}}(t) W_{k} d_{\epsilon} d x+\int_{(0, l)} \int_{\partial S} G_{\hat{i}}(t) W_{k} d_{\epsilon} \epsilon \sqrt{\nu_{i} o^{i j, \epsilon} \nu_{j}} d S d x_{1},
\end{gathered}
$$

for a.a. $t \in(0, T)$, completed with the initial states

$$
d_{\epsilon, k, \hat{i}}^{m}(0)=\int_{\Omega} \rho Q_{0, \epsilon, \hat{i}} W_{k} d_{\epsilon} d x, \quad \dot{d}_{\epsilon, k, \hat{i}}^{m}(0)=\int_{\Omega} \rho Q_{1, \epsilon, \hat{i}} W_{k} d_{\epsilon} d x
$$

for $j=1,2,3, k=1, \ldots, m, \hat{i}=1,2,3$.

Since the proof of the existence and uniqueness of a solution to the problem (5.6)-(5.7) is very close to the proofs in [5] or [6], we omit it.

Lemma 5.2. Under the assumptions of Section 4, the solution to the problem (5.6)-(5.7) satisfies the estimates

$$
\begin{aligned}
& \left\|\partial_{t} \mathbf{U}_{\epsilon}^{m}\right\|_{L^{\infty}\left(0, T ; L^{2}(\Omega)^{3}\right)}^{2}+\left\|\frac{1}{\epsilon} \omega\left(\mathbf{U}_{\epsilon}^{m}\right)\right\|_{L^{\infty}\left(0, T ; L^{2}(\Omega)^{9}\right)}^{2} \\
& \quad \leq C\left(\left\|\mathbf{Q}_{1, \epsilon}^{m}\right\|_{2}^{2}+\left\|\frac{1}{\epsilon} \omega\left(\mathbf{Q}_{0, \epsilon}^{m}\right)\right\|_{2}^{2}+\|\mathbf{F}\|_{L^{2}\left(0, T ; L^{2}(\Omega)^{3}\right)}^{2}+\|\mathbf{G}\|_{W^{1,1}\left(0, T ; L^{2}\left(0, l ; L^{2}(\partial S)^{3}\right)\right)}^{2}\right), \\
& \left\|\rho \partial_{t t} \mathbf{U}_{\epsilon}^{m}\right\|_{L^{2}\left(0, T ;\left[V(\Omega)^{3}\right]^{\prime}\right)}+\left\|\rho d_{\epsilon} \partial_{t t} \mathbf{U}_{\epsilon}^{m}\right\|_{L^{2}\left(0, T ;\left[V(\Omega)^{3}\right]^{\prime}\right)} \\
& \quad \leq C\left(\|\mathbf{F}\|_{L^{2}\left(0, T ; L^{2}(\Omega)^{3}\right)}+\|\mathbf{G}\|_{L^{2}\left(0, T ; L^{2}\left(0, l ; L^{2}(\partial S)^{3}\right)\right)}+\frac{1}{\epsilon^{2}}\left\|\omega^{\epsilon}\left(\mathbf{U}_{\epsilon}^{m}\right)\right\|_{L^{2}\left(0, T ; L^{2}(\Omega)^{9}\right)}\right),
\end{aligned}
$$

where the constant $C$ is independent of $\epsilon$. 
434 An asymptotic dynamic model for curved rods

Proof. We multiply (5.6) by $\dot{d}_{\epsilon, k, i}^{m}(t), \hat{i}=1,2,3$, sum $k=1, \ldots, m$ and recall (5.4)-(5.5) to discover (we do not use the summation convention for index $\hat{i}$ here) that

$$
\begin{array}{r}
\int_{\Omega} \rho \partial_{t t} U_{\epsilon, \hat{i}}^{m}(t) \partial_{t} U_{\epsilon, \hat{i}}^{m}(t) d_{\epsilon} d x+\int_{\Omega} A_{\epsilon}^{i j k l} \frac{1}{\epsilon} \omega_{k l}^{\epsilon}\left(\mathbf{U}_{\epsilon}^{m}(t)\right) \frac{1}{\epsilon} \omega_{i j}^{\epsilon}\left(\partial_{t} \hat{\mathbf{U}}_{\epsilon}^{m, \hat{i}}(t)\right) d_{\epsilon} d x \\
=\int_{\Omega} F_{\hat{i}}(t) \partial_{t} U_{\epsilon, \hat{i}}^{m}(t) d_{\epsilon} d x+\int_{0}^{l} \int_{\partial S} G_{\hat{i}}(t) \partial_{t} U_{\epsilon, \hat{i}}^{m}(t) d_{\epsilon} \epsilon \sqrt{v_{i} o^{i j, \epsilon} v_{j}} d S d x_{1},
\end{array}
$$

$\hat{i}=1,2,3$, where $\hat{\mathbf{U}}_{\epsilon}^{m, 1}=\left(U_{\epsilon, 1}^{m}, 0,0\right), \hat{\mathbf{U}}_{\epsilon}^{m, 2}=\left(0, U_{\epsilon, 2}^{m}, 0\right), \hat{\mathbf{U}}_{\epsilon}^{m, 3}=\left(0,0, U_{\epsilon, 3}^{m}\right)$. Summing $\hat{i}=$ $1,2,3$ in (5.10) and integrating (5.10) over the interval $[0, t], t \in(0, T)$, yield, together with (5.4)-(5.5) and (5.7),

$$
\begin{aligned}
\frac{1}{2}\left\|\sqrt{\rho d_{\epsilon}} \partial_{t} \mathbf{U}_{\epsilon}^{m}(t)\right\|_{2}^{2}+\frac{1}{2 \epsilon^{2}} \int_{\Omega} A_{\epsilon}^{i j k l} \omega_{k l}^{\epsilon}\left(\mathbf{U}_{\epsilon}^{m}(t)\right) \omega_{i j}^{\epsilon}\left(\mathbf{U}_{\epsilon}^{m}(t)\right) d_{\epsilon} d x \\
=\frac{1}{2}\left\|\sqrt{\rho d_{\epsilon}} \mathbf{Q}_{1, \epsilon}^{m}\right\|_{2}^{2} \\
\quad+\frac{1}{2 \epsilon^{2}} \int_{\Omega} A_{\epsilon}^{i j k l} \omega_{k l}^{\epsilon}\left(\mathbf{Q}_{0, \epsilon}^{m}\right) \omega_{i j}^{\epsilon}\left(\mathbf{Q}_{0, \epsilon}^{m}\right) d_{\epsilon} d x+\int_{0}^{t} \int_{\Omega}\left(\mathbf{F}(s), \partial_{t} \mathbf{U}_{\epsilon}^{m}(s)\right) d_{\epsilon} d x d s \\
+\int_{0}^{t} \int_{0}^{l} \int_{\partial S}\left(\mathbf{G}(s), \partial_{t} \mathbf{U}_{\epsilon}^{m}(s)\right) d_{\epsilon} \epsilon \sqrt{\nu_{i} o^{i j, \epsilon} v_{j}} d S d x_{1} d s
\end{aligned}
$$

for all $t \in[0, T]$. Further, we can estimate the third and fourth term on the right-hand side, using (3.11), (3.12) and the Young inequality, by

$$
\begin{aligned}
& \mid \int_{0}^{t} \int_{\Omega}\left(\mathbf{F}(s), \partial_{t} \mathbf{U}_{\epsilon}^{m}(s)\right) d_{\epsilon} d x d s \mid \leq \frac{C_{1}}{2 C_{7}^{2}} \int_{0}^{t}\|\mathbf{F}(s)\|_{2}^{2} d s+\frac{C_{1} C_{7}^{2}}{2} \int_{0}^{t}\left\|\partial_{t} \mathbf{U}_{\epsilon}^{m}(s)\right\|_{2}^{2} d s \\
&= \frac{C_{1}}{2 C_{7}^{2}}\|\mathbf{F}\|_{L^{2}\left(0, T ; L^{2}(\Omega)^{3}\right)}^{2}+T \frac{C_{1} C_{7}^{2}}{2}\left\|\partial_{t} \mathbf{U}_{\epsilon}^{m}(t)\right\|_{L^{\infty}\left(0, T ; L^{2}(\Omega)^{3}\right)}^{2} \\
&\left|\int_{0}^{t} \int_{0}^{l} \int_{\partial S}\left(\mathbf{G}(s), \partial_{t} \mathbf{U}_{\epsilon}^{m}(s)\right) d_{\epsilon} \epsilon \sqrt{\nu_{i} o^{i j, \epsilon} v_{j}} d S d x_{1} d s\right| \\
&=\mid \int_{0}^{l} \int_{\partial S}\left(\mathbf{G}(t), \mathbf{U}_{\epsilon}^{m}(t)\right) d_{\epsilon} \epsilon \sqrt{\nu_{i} o^{i j, \epsilon} v_{j}} d S d x_{1}-\int_{0}^{l} \int_{\partial S}\left(\mathbf{G}(0), \mathbf{Q}_{0, \epsilon}^{m}\right) d_{\epsilon} \epsilon \sqrt{v_{i} o^{i j, \epsilon} v_{j}} d S d x_{1} \\
& \quad-\int_{0}^{t} \int_{0}^{l} \int_{\partial S}\left(\partial_{t} \mathbf{G}(s), \mathbf{U}_{\epsilon}^{m}(s)\right) d_{\epsilon} \epsilon \sqrt{v_{i} o^{i j, \epsilon} \nu_{j}} d S d x_{1} d s \mid \\
& \leq \frac{C_{2}}{2 C_{8}^{2}}\|\mathbf{G}\|_{L^{\infty}\left(0, T ; L^{2}\left(0, l ; L^{2}(\partial S)^{3}\right)\right)}^{2}+\frac{C_{2} C_{8}^{2}}{2}\left\|\mathbf{U}_{\epsilon}^{m}\right\|_{L^{\infty}\left(0, T ; L^{2}\left(0, l ; L^{2}(\partial S)^{3}\right)\right)}^{2} \\
& \quad+\frac{C_{2}}{2 C_{9}^{2}}\|\mathbf{G}(0)\|_{L^{2}\left(0, l ; L^{2}(\partial S)^{3}\right)}^{2}+\frac{C_{2} C_{9}^{2}}{2}\left\|\mathbf{Q}_{0, \epsilon}^{m}\right\|_{L^{2}\left(0, l ; L^{2}(\partial S)^{3}\right)} \\
&+\frac{C_{2} C_{10}^{2}}{2}\left\|\mathbf{U}_{\epsilon}^{m}\right\|_{L^{\infty}\left(0, T ; L^{2}\left(0, l ; L^{2}(\partial S)^{3}\right)\right)}^{2}+\frac{C_{2}}{2 C_{10}^{2}}\left\|\partial_{t} \mathbf{G}\right\|_{L^{1}\left(0, T ; L^{2}\left(0, l ; L^{2}(\partial S)^{3}\right)\right)}^{2}
\end{aligned}
$$


for arbitrary positive constants $C_{7}, C_{8}, C_{9}, C_{10}$. Let constants $C_{11}, C_{12}$ come from the imbeddings

$$
W^{1,1}\left(0, T ; L^{2}\left(0, l ; L^{2}(\partial S)^{3}\right)\right) \hookrightarrow C\left([0, T] ; L^{2}\left(0, l ; L^{2}(\partial S)^{3}\right)\right), \quad H^{1}(S) \smile L^{2}(\partial S),
$$

respectively. The estimate $\left\|\left(A_{\epsilon}^{i j k l}\right)_{i, j, k, l=1}^{3}\right\|_{C(\bar{\Omega})} \leq C_{13}$ holds with the constant $C_{13}$ being independent of $\epsilon$ as a consequence of the relations (2.6), (2.18)-(2.19) and (3.5). Hence, from (4.10), from the estimates (3.6), (3.7) and (5.11)-(5.13), it follows that

$$
\begin{aligned}
\left(\frac{C_{0} C_{5}}{2}-\right. & \left.T \frac{C_{1} C_{7}^{2}}{2}\right)\left\|\partial_{t} \mathbf{U}_{\epsilon}^{m}\right\|_{L^{\infty}\left(0, T ; L^{2}(\Omega)^{3}\right)}^{2} \\
& +\frac{C_{0}-C_{2} C_{3} C_{4}^{2} C_{12}^{2}\left(C_{8}^{2}+C_{10}^{2}\right)}{2 \epsilon^{2} C_{3}}\left\|\omega^{\epsilon}\left(\mathbf{U}_{\epsilon}^{m}\right)\right\|_{L^{\infty}\left(0, T ; L^{2}(\Omega)^{9}\right)}^{2} \\
\leq & \frac{C_{1} C_{6}}{2}\left\|\mathbf{Q}_{1, \epsilon}^{m}\right\|_{2}^{2}+\frac{C_{1} C_{13}+C_{2} C_{4}^{2} C_{9}^{2} C_{12}^{2}}{2 \epsilon^{2}}\left\|\omega^{\epsilon}\left(\mathbf{Q}_{0, \epsilon}^{m}\right)\right\|_{2}^{2}+\frac{C_{1}}{2 C_{7}^{2}}\|\mathbf{F}\|_{L^{2}\left(0, T ; L^{2}(\Omega)^{3}\right)}^{2} \\
& +C_{2}\left(\frac{C_{11}^{2}}{2 C_{8}^{2}}+\frac{C_{11}^{2}}{2 C_{9}^{2}}+\frac{1}{2 C_{10}^{2}}\right)\|\mathbf{G}\|_{W^{1,1}\left(0, T ; L^{2}\left(0, l ; L^{2}(\partial S)^{3}\right)\right)^{2}}^{2}
\end{aligned}
$$

Putting now $C_{7}=\sqrt{C_{0} C_{5} / 2 T C_{1}}, C_{10}=C_{8}, C_{8}=\left(C_{0} / 4 C_{2} C_{3} C_{4}^{2} C_{12}^{2}\right)^{1 / 4}$, we obtain (5.8).

It remains to show (5.9). We fix any $V \in V(\Omega)$ such that $\|V\|_{1,2} \leq 1$. The function $V / d_{\epsilon}$ belongs to $V(\Omega)$ for $\epsilon$ sufficiently small as well, which is a consequence of (2.5), (2.13), (2.20) and (3.11). In addition, $\left\|V / d_{\epsilon}\right\|_{1,2} \leq C_{14}$, where the constant $C_{14}$ is independent of $\epsilon$. We can decompose this function as a sum $V / d_{\epsilon}=V_{1}^{\epsilon}+V_{2}^{\epsilon}$, where $V_{1}^{\epsilon} \in \operatorname{span}\left\{W_{k}\right\}_{k=1}^{m}$ and $\left(\left(V_{2}^{\epsilon}, W_{k}\right)\right)_{\rho d_{\epsilon}, \Omega}=0, k=1, \ldots, m$. We can derive from (3.11) and (4.10) the estimate

$$
\|V\|_{1,2}^{2} \geq \frac{C_{0} C_{5}}{C_{1} C_{6}}\left\|V_{1}^{\epsilon}\right\|_{1,2}
$$

and thus $\left\|V_{1}^{\epsilon}\right\|_{1,2} \leq C_{15}=C_{14} C_{1} C_{6} / C_{0} C_{5}$, where $C_{15}$ is independent of $\epsilon$. Then (5.4)-(5.6) imply, after substitution $W_{k}=V_{1}^{\epsilon}$, that

$$
\begin{aligned}
\int_{\Omega} \rho \partial_{t t} & U_{\epsilon, \hat{i}}^{m}(t) \frac{V}{d_{\epsilon}} d_{\epsilon} d x \\
= & \int_{\Omega} \rho \partial_{t t} U_{\epsilon, \hat{i}}^{m}(t) V_{1}^{\epsilon} d_{\epsilon} d x \\
= & \int_{\Omega} F_{\hat{i}}(t) V_{1}^{\epsilon} d_{\epsilon} d x+\int_{0}^{l} \int_{\partial S} G_{\hat{i}}(t) V_{1}^{\epsilon} d_{\epsilon} \epsilon \sqrt{\nu_{i} o^{i j, \epsilon} \nu_{j}} d S d x_{1} \\
& -\int_{\Omega} A_{\epsilon}^{i j k l} \frac{1}{\epsilon} \omega_{k l}^{\epsilon}\left(\mathbf{U}_{\epsilon}^{m}(t)\right) \frac{1}{\epsilon} \omega_{i j}^{\epsilon}\left(\mathbf{V}_{1}^{\hat{i}, \epsilon}\right) d_{\epsilon} d x,
\end{aligned}
$$

$\hat{i}=1,2,3$, where $\mathbf{V}_{1}^{1, \epsilon}=\left(V_{1}^{\epsilon}, 0,0\right), \mathbf{V}_{1}^{2, \epsilon}=\left(0, V_{1}^{\epsilon}, 0\right), \mathbf{V}_{1}^{3, \epsilon}=\left(0,0, V_{1}^{\epsilon}\right)$. We can see that the estimate

$$
\int_{\Omega} A_{\epsilon}^{i j k l} \frac{1}{\epsilon} \omega_{k l}^{\epsilon}\left(\mathbf{U}_{\epsilon}^{m}(t)\right) \frac{1}{\epsilon} \omega_{i j}^{\epsilon}\left(\mathbf{V}_{1}^{\hat{i}, \epsilon}\right) d_{\epsilon} d x \leq \frac{C}{\epsilon^{2}}\left\|\omega^{\epsilon}\left(\mathbf{U}_{\epsilon}^{m}(t)\right)\right\|_{2}
$$


holds for a.a. $t \in(0, T)$, where the constant $C$ is independent of $\epsilon$. Hence

$$
\begin{aligned}
& \left|\sum_{\hat{i}=1}^{3}[V(\Omega)]^{\prime}\left\langle\rho \partial_{t t} U_{\epsilon, \hat{i}}^{m}(t), V\right\rangle_{V(\Omega)}\right| \\
& \quad \leq C\left(\|\mathbf{F}(t)\|_{2}+\|\mathbf{G}(t)\|_{L^{2}\left(0, l ; L^{2}(\partial S)^{3}\right)}+\frac{1}{\epsilon^{2}}\left\|\omega^{\epsilon}\left(\mathbf{U}_{\epsilon}^{m}(t)\right)\right\|_{2}\right), \quad \text { for a.a. } t \in(0, T),
\end{aligned}
$$

where the constant $C$ is independent of $\epsilon$. Taking the function $V$ instead of $V / d_{\epsilon}$, and using the same procedure as above for the term $\rho d_{\epsilon} \partial_{t t} \mathbf{U}_{\epsilon}^{m}$, we get (5.9).

Proof of Proposition 5.1. Using (4.12), (5.4), (5.5), (5.7), we can easily derive that

$$
\mathbf{Q}_{0, \epsilon}^{m} \longrightarrow \mathbf{Q}_{0, \epsilon} \quad \text { in } V(\Omega)^{3}, \quad \mathbf{Q}_{1, \epsilon}^{m} \longrightarrow \mathbf{Q}_{1, \epsilon} \quad \text { in } L^{2}(\Omega)^{3}
$$

From the estimates (3.7) and (5.8), it follows (passing to a subsequence if necessary) that

$$
\begin{array}{r}
\mathbf{U}_{\epsilon}^{m} \stackrel{*}{\longrightarrow} \mathbf{U}_{\epsilon} \quad \text { in } L^{\infty}\left(0, T ; H^{1}(\Omega)^{3}\right), \\
\partial_{t} \mathbf{U}_{\epsilon}^{m} \stackrel{*}{\rightarrow} \partial_{t} \mathbf{U}_{\epsilon} \quad \text { in } L^{\infty}\left(0, T ; L^{2}(\Omega)^{3}\right), \\
\rho \partial_{t} \mathbf{U}_{\epsilon}^{m} \stackrel{*}{\rightarrow} \rho \partial_{t} \mathbf{U}_{\epsilon} \quad \text { in } L^{\infty}\left(0, T ; L^{2}(\Omega)^{3}\right), \\
\rho \partial_{t t} \mathbf{U}_{\epsilon}^{m} \longrightarrow \mathbf{W}_{\epsilon} \quad \text { in } L^{2}\left(0, T ; H^{-1}(\Omega)^{3}\right), \\
\rho d_{\epsilon} \partial_{t t} \mathbf{U}_{\epsilon}^{m} \longrightarrow \widehat{\mathbf{W}}_{\epsilon} \quad \text { in } L^{2}\left(0, T ; H^{-1}(\Omega)^{3}\right)
\end{array}
$$

for $m \rightarrow \infty$. It remains to show that $\mathbf{W}_{\epsilon}=\rho \partial_{t t} \mathbf{U}_{\epsilon}$ and $\widehat{\mathbf{W}}_{\epsilon}=\rho d_{\epsilon} \partial_{t t} \mathbf{U}_{\epsilon}$. From (5.23), it follows that

$$
\rho \partial_{t t} \mathbf{U}_{\epsilon}^{m} \longrightarrow \rho \partial_{t t} \mathbf{U}_{\epsilon}, \quad \rho d_{\epsilon} \partial_{t t} \mathbf{U}_{\epsilon}^{m} \longrightarrow \rho d_{\epsilon} \partial_{t t} \mathbf{U}_{\epsilon}, \quad \text { in } W^{-1,2}\left(0, T ; L^{2}(\Omega)^{3}\right)
$$

for $m \rightarrow \infty$, which leads to the desired conclusion. The estimates (5.1)-(5.2) immediately follow from (5.8)-(5.9) and (5.20)-(5.25). Using the standard theorems about compact imbeddings in Bochner's spaces (see [11]) together with (5.21)-(5.25), we can deduce that

$$
\begin{gathered}
\mathbf{U}_{\epsilon}^{m} \longrightarrow \mathbf{U}_{\epsilon} \quad \text { in } C^{\infty}\left([0, T] ; L^{2}(\Omega)^{3}\right), \\
\partial_{t} \mathbf{U}_{\epsilon}^{m} \longrightarrow \partial_{t} \mathbf{U}_{\epsilon} \quad \text { in } C\left([0, T] ; L_{\text {weak }}^{2}(\Omega)^{3}\right)
\end{gathered}
$$

for $m \rightarrow \infty$. The uniqueness of the solution follows from the linearity of (4.13) and from the estimate (5.1). 
Corollary 5.3. Under the assumptions of Proposition 5.1, there exists a sequence $\left\{\epsilon_{n}\right\}_{n=1}^{\infty}$ $\subset(0,1)$ such that $\epsilon_{n} \rightarrow 0$ and

$$
\begin{gathered}
\mathbf{U}_{\epsilon_{n}} \stackrel{*}{\rightarrow} \mathbf{U} \quad \text { in } L^{\infty}\left(0, T ; V(\Omega)^{3}\right), \\
\partial_{t} \mathbf{U}_{\epsilon_{n}} \stackrel{*}{\longrightarrow} \partial_{t} \mathbf{U} \quad \text { in } L^{\infty}\left(0, T ; L^{2}(\Omega)^{3}\right), \\
\frac{1}{\epsilon_{n}} \omega^{\epsilon_{n}}\left(\mathbf{U}_{\epsilon_{n}}\right) \stackrel{*}{\longrightarrow} \zeta \quad \text { in } L^{\infty}\left(0, T ; L^{2}(\Omega)^{9}\right)
\end{gathered}
$$

for $\epsilon_{n} \rightarrow 0$ and thus

$$
\begin{gathered}
\overline{\mathbf{U}}_{\epsilon_{n}}^{\varphi} \longrightarrow \overline{\mathbf{U}}^{\varphi} \quad \text { in } H^{1}(\Omega)^{3}, \\
{\bar{\partial} \mathbf{U}_{\epsilon_{n}}}^{\varphi} \longrightarrow \bar{\partial}_{t}^{\varphi}{ }^{\varphi} \quad \text { in } L^{2}(\Omega)^{3}, \\
\frac{1}{\epsilon_{n}} \omega^{\epsilon_{n}}\left({\overline{\mathbf{U}_{\epsilon_{n}}}}^{\varphi}\right)=\frac{1}{\epsilon_{n}} \bar{\omega}^{\epsilon_{n}\left(\mathbf{U}_{\epsilon_{n}}\right)} \longrightarrow \bar{\zeta}^{\varphi} \quad \text { in } L^{2}(\Omega)^{9},
\end{gathered}
$$

for all $\varphi \in C_{0}^{\infty}(0, T)$.

\section{Qualitative properties of the limit displacements}

Proposition 6.1. Suppose that $\left\{\epsilon_{n}\right\}_{n=1}^{\infty} \subset(0,1)$ and $\epsilon_{n} \rightarrow 0$. Let, in addition, a sequence $\left\{\mathbf{U}_{\epsilon_{n}}\right\}_{n=1}^{\infty} \subset L^{\infty}\left(0, T ; V(\Omega)^{3}\right)$ be such that

$$
\begin{gathered}
\mathbf{U}_{\epsilon_{n}} \stackrel{*}{\longrightarrow} \mathbf{U} \quad \text { in } L^{\infty}\left(0, T ; V(\Omega)^{3}\right), \\
\frac{1}{\epsilon_{n}} \omega^{\epsilon_{n}}\left(\mathbf{U}_{\epsilon_{n}}\right) \stackrel{*}{\longrightarrow} \zeta \quad \text { in } L^{\infty}\left(0, T ; L^{2}(\Omega)^{9}\right),
\end{gathered}
$$

for $\epsilon_{n} \rightarrow 0$. Then the couple $\lfloor\mathbf{U}, \phi\rfloor \in L^{\infty}\left(0, T ; \mathscr{V}_{0}^{\mathbf{t}, \mathbf{n}, \mathbf{b}}(0, l)\right)$ (in the sense $\partial_{j} \mathbf{U}=0, j=2,3$ ), where the function $\phi$ is such that

$$
\frac{1}{2 \epsilon_{n}}\left(\left(\partial_{2} \mathbf{U}_{\epsilon_{n}}, \mathbf{b}_{\epsilon_{n}}\right)-\left(\partial_{3} \mathbf{U}_{\epsilon_{n}}, \mathbf{n}_{\epsilon_{n}}\right)\right) \stackrel{*}{\longrightarrow} \phi
$$

in $L^{\infty}\left(0, T ; L^{2}(\Omega)\right)$ for $\epsilon_{n} \rightarrow 0$. In addition, the couple $\lfloor\mathbf{U}, \phi\rfloor$ generates a function $\mathbf{U}_{*} \in$ $L^{\infty}\left(0, T ; H_{0}^{1}(0, l)^{3}\right)$ which together with the function $\mathbf{U}$ satisfies the relations

$$
\begin{aligned}
\left(\partial_{1} \mathbf{U}, \mathbf{t}\right) & =0 \quad \text { a.e.in }(0, l) \times(0, T), \\
\left(\partial_{1} \mathbf{U}_{*}, \mathbf{t}\right) & =\partial_{3} \zeta_{12}-\partial_{2} \zeta_{13} \quad \text { in } L^{\infty}\left(0, T ; L^{2}\left(0, l ; H^{-1}(S)\right)\right), \\
\left(\partial_{1} \mathbf{U}_{*}, \mathbf{n}\right) & =-\partial_{3} \zeta_{11} \quad \text { a.e. in }(0, l) \times(0, T), \\
\left(\partial_{1} \mathbf{U}_{*}, \mathbf{b}\right) & =\partial_{2} \zeta_{11} \quad \text { a.e. in }(0, l) \times(0, T) .
\end{aligned}
$$

Remark 6.2. Since $\overline{\left(1 / \epsilon_{n}\right) \omega^{\epsilon_{n}}\left(\mathbf{U}_{\epsilon_{n}}\right)}{ }^{\varphi}=\left(1 / \epsilon_{n}\right) \omega^{\epsilon_{n}}\left({\overline{\mathbf{U}_{\epsilon_{n}}}}^{\varphi}\right)$ (see (4.6)-(4.9)), we can use (5.31), (5.33) and [14, Proposition 7.2] to derive the existence of the pair $\left\lfloor\overline{\mathbf{U}}^{\varphi}, \phi_{\varphi}\right\rfloor \in \mathscr{V}_{0}^{\mathbf{t}, \mathbf{n}, \mathbf{b}}(0$, l) (in the sense $\partial_{j} \overline{\mathbf{U}}^{\varphi}=0, j=2,3$ ) for arbitrary $\varphi \in C_{0}^{\infty}(0, T)$, where the function $\phi_{\varphi}$ is 
438 An asymptotic dynamic model for curved rods

such that

$$
\frac{1}{2 \epsilon_{n}}\left(\left(\partial_{2}{\overline{\mathbf{U}_{\epsilon_{n}}}}^{\varphi}, \mathbf{b}_{\epsilon_{n}}\right)-\left(\partial_{3}{\overline{\mathbf{U}_{\epsilon_{n}}}}^{\varphi}, \mathbf{n}_{\epsilon_{n}}\right)\right) \longrightarrow \phi_{\varphi}
$$

in $L^{2}(\Omega)$ for $\epsilon_{n} \rightarrow 0$ and for arbitrary $\varphi \in C_{0}^{\infty}(0, T)$. In addition, the couple $\left\lfloor\overline{\mathbf{U}}^{\varphi}, \phi_{\varphi}\right\rfloor$ generates the function $\mathbf{U}_{*, \varphi} \in H_{0}^{1}(0, l)^{3}$ which together with the function $\overline{\mathbf{U}}^{\varphi}$ satisfies the relations

$$
\begin{aligned}
\left(\partial_{1} \overline{\mathbf{U}}^{\varphi}, \mathbf{t}\right) & =0 \quad \text { a.e. in }(0, l), \\
\left(\partial_{1} \mathbf{U}_{*, \varphi}, \mathbf{t}\right) & =\bar{\partial}_{3} \zeta_{12}-\partial_{2} \zeta_{13} \\
\left(\partial_{1} \mathbf{U}_{*, \varphi}, \mathbf{n}\right) & ={\overline{-\partial_{3} \zeta_{11}}}^{\varphi} \quad \text { in } L^{2}\left(0, l ; H^{-1}(S)\right), \\
\left(\partial_{1} \mathbf{U}_{*, \varphi}, \mathbf{b}\right) & ={\bar{\partial} \zeta_{11}}^{\varphi} \quad \text { a.e. in }(0, l),
\end{aligned}
$$

for arbitrary $\varphi \in C_{0}^{\infty}(0, T)$. If the sequence $\left\{\left(1 / \epsilon_{n}\right) \omega^{\epsilon_{n}}\left({\overline{\mathbf{U}_{\epsilon_{n}}}}^{\varphi}\right)\right\}_{n=1}^{\infty}$ converges strongly in $L^{2}(\Omega)^{9}$, the convergence of the sequence $\left\{\overline{\mathbf{U}}_{\epsilon_{n}}{ }^{\varphi}\right\}_{n=1}^{\infty}$ is strong as well for arbitrary $\varphi \in$ $C_{0}^{\infty}(0, T)$.

Remark 6.3. From Remark 6.2, it follows that to prove Proposition 6.1, we must check that

$$
\phi_{\varphi}\left(x_{1}\right)=\bar{\phi}^{\varphi}\left(x_{1}\right), \quad \mathbf{U}_{*, \varphi}\left(x_{1}\right)={\overline{\mathbf{U}_{*}}}^{\varphi}\left(x_{1}\right)
$$

for all $\varphi \in C_{0}^{\infty}(0, T)$ and for a.a. $x_{1} \in(0, l)$, which is the main task of this section.

We define auxiliary functions $\phi_{\epsilon}, \epsilon \in(0,1)$, by the relation

$$
\phi_{\epsilon}=\frac{1}{2 \epsilon}\left(\left(\partial_{2} \mathbf{U}_{\epsilon}, \mathbf{b}_{\epsilon}\right)-\left(\partial_{3} \mathbf{U}_{\epsilon}, \mathbf{n}_{\epsilon}\right)\right) .
$$

Further, we define the vector functions $\mathbf{U}_{*, \epsilon}, \epsilon \in(0,1)$, by

$$
\mathbf{U}_{*, \epsilon}=-\phi_{\epsilon} \mathbf{t}_{\epsilon}-\frac{1}{\epsilon}\left(\partial_{3} \mathbf{U}_{\epsilon}, \mathbf{g}_{1, \epsilon}\right) \mathbf{n}_{\epsilon}+\frac{1}{\epsilon}\left(\partial_{2} \mathbf{U}_{\epsilon}, \mathbf{g}_{1, \epsilon}\right) \mathbf{b}_{\epsilon}
$$

Proof of Proposition 6.1. To simplify the notation, we will use $\epsilon$ instead of $\epsilon_{n}$. Using (2.5), (2.16), (4.6)-(4.9) and (6.15), we can derive the expressions

$$
\begin{aligned}
\left(\partial_{1} \mathbf{U}_{*, \epsilon}, \mathbf{t}_{\epsilon}\right)= & \frac{1}{\epsilon^{2}} \partial_{3} \theta_{12}^{\epsilon}\left(\mathbf{U}_{\epsilon}\right)+\frac{1}{\epsilon} \partial_{3} \kappa_{12}^{\epsilon}\left(\mathbf{U}_{\epsilon}\right)-\frac{1}{\epsilon^{2}} \partial_{2} \theta_{13}^{\epsilon}\left(\mathbf{U}_{\epsilon}\right)-\frac{1}{\epsilon} \partial_{2} \kappa_{13}^{\epsilon}\left(\mathbf{U}_{\epsilon}\right) \\
& -\left(\beta_{\epsilon}^{2} x_{2}+\alpha_{\epsilon} \beta_{\epsilon} x_{3}\right)\left(\partial_{3} \mathbf{U}_{\epsilon}, \mathbf{t}_{\epsilon}\right)+\left(\alpha_{\epsilon} \beta_{\epsilon} x_{2}+\alpha_{\epsilon}^{2} x_{3}\right)\left(\partial_{2} \mathbf{U}_{\epsilon}, \mathbf{t}_{\epsilon}\right) \\
& -\left(\beta_{\epsilon} \gamma_{\epsilon} x_{2}+\frac{\gamma_{\epsilon}}{\epsilon}\right)\left(\partial_{3} \mathbf{U}_{\epsilon}, \mathbf{b}_{\epsilon}\right) \\
& -\left(\alpha_{\epsilon} \gamma_{\epsilon} x_{3}+\frac{\gamma_{\epsilon}}{\epsilon}\right)\left(\partial_{2} \mathbf{U}_{\epsilon}, \mathbf{n}_{\epsilon}\right)+\beta_{\epsilon} \gamma_{\epsilon} x_{3}\left(\partial_{3} \mathbf{U}_{\epsilon}, \mathbf{n}_{\epsilon}\right)+\alpha_{\epsilon} \gamma_{\epsilon} x_{2}\left(\partial_{2} \mathbf{U}_{\epsilon}, \mathbf{b}_{\epsilon}\right),
\end{aligned}
$$




$$
\begin{aligned}
\left(\partial_{1} \mathbf{U}_{*, \epsilon}, \mathbf{n}_{\epsilon}\right)= & -\frac{\partial_{3} \kappa_{11}^{\epsilon}\left(\mathbf{U}_{\epsilon}\right)}{\epsilon}+\frac{\beta_{\epsilon}}{\epsilon}\left(\frac{\left(\partial_{2} \mathbf{U}_{\epsilon}, \mathbf{b}_{\epsilon}\right)+\left(\partial_{3} \mathbf{U}_{\epsilon}, \mathbf{n}_{\epsilon}\right)}{2}\right) \\
& +\gamma_{\epsilon}\left(\frac{1}{\epsilon}\left(\partial_{2} \mathbf{U}_{\epsilon}, \mathbf{g}_{1, \epsilon}\right)+\left(\partial_{1} \mathbf{U}_{\epsilon}, \mathbf{n}_{\epsilon}\right)\right) \\
& +\left(-\frac{\alpha_{\epsilon}}{\epsilon}+\alpha_{\epsilon}^{2} x_{3}+\alpha_{\epsilon} \beta_{\epsilon} x_{2}+\gamma_{\epsilon}^{\prime} x_{2}+\gamma_{\epsilon}^{2} x_{3}\right)\left(\partial_{3} \mathbf{U}_{\epsilon}, \mathbf{b}_{\epsilon}\right) \\
& +\left(\beta_{\epsilon}^{\prime} x_{2}+\alpha_{\epsilon}^{\prime} x_{3}+\beta_{\epsilon} \gamma_{\epsilon} x_{3}-\alpha_{\epsilon} \gamma_{\epsilon} x_{2}\right)\left(\partial_{3} \mathbf{U}_{\epsilon}, \mathbf{t}_{\epsilon}\right) \\
& +\left(\alpha_{\epsilon} \beta_{\epsilon} x_{3}+\beta_{\epsilon}^{2} x_{2}+\gamma_{\epsilon}^{2} x_{2}-\gamma_{\epsilon}^{\prime} x_{3}\right)\left(\partial_{3} \mathbf{U}_{\epsilon}, \mathbf{n}_{\epsilon}\right)-\alpha_{\epsilon}\left(\partial_{1} \mathbf{U}_{\epsilon}, \mathbf{t}_{\epsilon}\right), \\
\left(\partial_{1} \mathbf{U}_{*, \epsilon}, \mathbf{b}_{\epsilon}\right)= & \frac{\partial_{2} \kappa_{11}^{\epsilon}\left(\mathbf{U}_{\epsilon}\right)}{\epsilon}+\frac{\alpha_{\epsilon}}{\epsilon}\left(\frac{\left(\partial_{2} \mathbf{U}_{\epsilon}, \mathbf{b}_{\epsilon}\right)+\left(\partial_{3} \mathbf{U}_{\epsilon}, \mathbf{n}_{\epsilon}\right)}{2}\right) \\
& +\gamma_{\epsilon}\left(\frac{1}{\epsilon}\left(\partial_{3} \mathbf{U}_{\epsilon}, \mathbf{g}_{1, \epsilon}\right)+\left(\partial_{1} \mathbf{U}_{\epsilon}, \mathbf{b}_{\epsilon}\right)\right) \\
& -\left(-\frac{\beta_{\epsilon}}{\epsilon}+\beta_{\epsilon}^{2} x_{2}+\alpha_{\epsilon} \beta_{\epsilon} x_{3}-\gamma_{\epsilon}^{\prime} x_{3}+\gamma_{\epsilon}^{2} x_{2}\right)\left(\partial_{2} \mathbf{U}_{\epsilon}, \mathbf{n}_{\epsilon}\right) \\
& -\left(\beta_{\epsilon}^{\prime} x_{2}+\alpha_{\epsilon}^{\prime} x_{3}+\beta_{\epsilon} \gamma_{\epsilon} x_{3}-\alpha_{\epsilon} \gamma_{\epsilon} x_{2}\right)\left(\partial_{2} \mathbf{U}_{\epsilon}, \mathbf{t}_{\epsilon}\right) \\
& -\left(\alpha_{\epsilon} \beta_{\epsilon} x_{2}+\alpha_{\epsilon}^{2} x_{3}+\gamma_{\epsilon}^{2} x_{3}+\gamma_{\epsilon}^{\prime} x_{2}\right)\left(\partial_{2} \mathbf{U}_{\epsilon}, \mathbf{b}_{\epsilon}\right)-\beta_{\epsilon}\left(\partial_{1} \mathbf{U}_{\epsilon}, \mathbf{t}_{\epsilon}\right)
\end{aligned}
$$

in the sense of the space $L^{\infty}\left(0, T ; L^{2}\left(0, l ; H^{-1}(S)\right)\right)$. Since the estimate

$$
\begin{aligned}
&\left\|\frac{1}{\epsilon^{2 r}}\left(\partial_{3} \mathbf{U}_{\epsilon}(t), \mathbf{n}_{\epsilon}\right)\right\|_{2}+\left\|\frac{1}{\epsilon^{2 r}}\left(\partial_{2} \mathbf{U}_{\epsilon}(t), \mathbf{b}_{\epsilon}\right)\right\|_{2} \\
& \leq\left\|\frac{1}{\epsilon^{2 r}}\left(\left(\partial_{3} \mathbf{U}_{\epsilon}(t), \mathbf{n}_{\epsilon}\right)+\left(\partial_{2} \mathbf{U}_{\epsilon}(t), \mathbf{b}_{\epsilon}\right)\right)\right\|_{2}+C \epsilon^{1-2 r} \sum_{j=2}^{3}\left\|\partial_{j} \phi_{\epsilon}(t)\right\|_{L^{2}\left(0, l ; H^{-1}(S)\right)} \\
&+\frac{C}{\epsilon^{2 r}}\left(\left\|\frac{1}{\epsilon} \partial_{3} \theta_{12}^{\epsilon}\left(\mathbf{U}_{\epsilon}(t)\right)+\partial_{3} \kappa_{12}^{\epsilon}\left(\mathbf{U}_{\epsilon}(t)\right)\right\|_{L^{2}\left(0, l ; H^{-1}(S)\right)}\right) \\
&\left.+\left\|\frac{1}{\epsilon} \partial_{2} \theta_{13}^{\epsilon}\left(\mathbf{U}_{\epsilon}(t)\right)+\partial_{2} \kappa_{13}^{\epsilon}\left(\mathbf{U}_{\epsilon}(t)\right)\right\|_{L^{2}\left(0, l ; H^{-1}(S)\right)}\right) \\
&+\frac{C}{\epsilon^{3 r}}\left(\left\|\left(\partial_{3} \mathbf{U}_{\epsilon}(t), \mathbf{g}_{1, \epsilon}\right)\right\|_{2}+\left\|\left(\partial_{2} \mathbf{U}_{\epsilon}(t), \mathbf{g}_{1, \epsilon}\right)\right\|_{2}\right) \\
&+C \epsilon^{1-4 r}\left(\left\|\left(\partial_{3} \mathbf{U}_{\epsilon}(t), \mathbf{t}_{\epsilon}\right)\right\|_{2}+\left\|\left(\partial_{2} \mathbf{U}_{\epsilon}(t), \mathbf{t}_{\epsilon}\right)\right\|_{2}\right) \\
&+\frac{C}{\epsilon^{3 r}}\left(\left\|\left(\partial_{3} \mathbf{U}_{\epsilon}(t), \mathbf{b}_{\epsilon}\right)\right\|_{2}+\left\|\left(\partial_{2} \mathbf{U}_{\epsilon}(t), \mathbf{n}_{\epsilon}\right)\right\|_{2}\right) \\
&+C \epsilon^{1-2 r}\left(\left\|\frac{1}{\epsilon^{2 r}}\left(\partial_{2} \mathbf{U}_{\epsilon}(t), \mathbf{b}_{\epsilon}\right)\right\|_{2}+\left\|\frac{1}{\epsilon^{2 r}}\left(\partial_{3} \mathbf{U}_{\epsilon}(t), \mathbf{n}_{\epsilon}\right)\right\|_{2}\right)
\end{aligned}
$$

holds for a.a. $t \in(0, T)$ (see [14]) and

$$
\begin{aligned}
& \partial_{2} \phi_{\epsilon}(t)=\frac{1}{2 \epsilon}\left(\partial_{2}\left(\partial_{2} \mathbf{U}_{\epsilon}(t), \mathbf{b}_{\epsilon}\right)+\partial_{2}\left(\partial_{3} \mathbf{U}_{\epsilon}(t), \mathbf{n}_{\epsilon}\right)\right)-\frac{1}{\epsilon} \partial_{3}\left(\partial_{2} \mathbf{U}_{\epsilon}(t), \mathbf{n}_{\epsilon}\right), \\
& \partial_{3} \phi_{\epsilon}(t)=\frac{1}{2 \epsilon}\left(\partial_{3}\left(\partial_{2} \mathbf{U}_{\epsilon}(t), \mathbf{b}_{\epsilon}\right)+\partial_{3}\left(\partial_{3} \mathbf{U}_{\epsilon}(t), \mathbf{n}_{\epsilon}\right)\right)-\frac{1}{\epsilon} \partial_{2}\left(\partial_{3} \mathbf{U}_{\epsilon}(t), \mathbf{b}_{\epsilon}\right)
\end{aligned}
$$


in $L^{2}\left(0, l ; H^{-1}(S)\right)$ for a.a. $t \in(0, T)$, we get from (2.6) and (6.2) *-weak convergence of the functions $\left(\partial_{1} \mathbf{U}_{*, \epsilon}, \mathbf{t}_{\epsilon}\right),\left(\partial_{1} \mathbf{U}_{*, \epsilon}, \mathbf{n}_{\epsilon}\right),\left(\partial_{1} \mathbf{U}_{*, \epsilon}, \mathbf{b}_{\epsilon}\right)$ in the space $L^{\infty}\left(0, T ; L^{2}\left(0, l ; H^{-1}(S)\right)\right)$, where the limit states correspond to the right-hand sides in (6.5)-(6.7), which is a consequence of Remark 6.2. Since

$$
\partial_{1} \mathbf{U}_{*, \epsilon}=\left(\partial_{1} \mathbf{U}_{*, \epsilon}, \mathbf{t}_{\epsilon}\right) \mathbf{t}_{\epsilon}+\left(\partial_{1} \mathbf{U}_{*, \epsilon}, \mathbf{n}_{\epsilon}\right) \mathbf{n}_{\epsilon}+\left(\partial_{1} \mathbf{U}_{*, \epsilon}, \mathbf{b}_{\epsilon}\right) \mathbf{b}_{\epsilon},
$$

we can easily derive from (2.2) that

$$
\partial_{1} \mathbf{U}_{*, \epsilon} \stackrel{*}{\longrightarrow}\left(\partial_{3} \zeta_{12}-\partial_{2} \zeta_{13}\right) \mathbf{t}-\partial_{3} \zeta_{11} \mathbf{n}+\partial_{2} \zeta_{11} \mathbf{b}
$$

in $L^{\infty}\left(0, T ; L^{2}\left(0, l ; H^{-1}(S)^{3}\right)\right)$.

Further, we want to prove that

$$
\partial_{j} \mathbf{U}_{*, \epsilon} \stackrel{*}{\longrightarrow} \mathbf{0} \quad \text { in } L^{\infty}\left(0, T ; L^{2}\left(0, l ; H^{-1}(S)^{3}\right)\right), j=2,3,
$$

and $\left.\mathbf{U}_{*, \epsilon}(t)\right|_{x_{1}=0}=\left.\mathbf{U}_{*, \epsilon}(t)\right|_{x_{1}=l}=\mathbf{0}$ for almost all $t \in(0, T)$ in the sense of the space $C([0, l]$; $\left.H^{-1}(S)^{3}\right)$.

If we fix $t \in(0, T)$, we can prove the second part of the above assertion in the same way as in [14]. The proof of (6.23) follows from the expression

$$
\begin{aligned}
\partial_{j} \mathbf{U}_{*, \epsilon}= & -\partial_{j} \phi_{\epsilon} \mathbf{t}_{\epsilon}+\partial_{j}\left(\partial_{1} \mathbf{U}_{\epsilon}, \mathbf{b}_{\epsilon}\right) \mathbf{n}_{\epsilon}-\partial_{j}\left(\partial_{1} \mathbf{U}_{\epsilon}, \mathbf{n}_{\epsilon}\right) \mathbf{b}_{\epsilon} \\
& -\partial_{j}\left(\frac{1}{\epsilon}\left(\partial_{3} \mathbf{U}_{\epsilon}, \mathbf{g}_{1, \epsilon}\right)+\left(\partial_{1} \mathbf{U}_{\epsilon}, \mathbf{b}_{\epsilon}\right)\right) \mathbf{n}_{\epsilon}+\partial_{j}\left(\frac{1}{\epsilon}\left(\partial_{2} \mathbf{U}_{\epsilon}, \mathbf{g}_{1, \epsilon}\right)+\left(\partial_{1} \mathbf{U}_{\epsilon}, \mathbf{n}_{\epsilon}\right)\right) \mathbf{b}_{\epsilon},
\end{aligned}
$$

from (4.6)-(4.9), (6.2) and from the fact that the function $\mathbf{U}$ depends only on $t$ and $x_{1}$ (see Remark 6.2). Applying Proposition 3.4 together with (6.22)-(6.23), we get

$$
\begin{gathered}
\partial_{j}{\overline{\mathbf{U}_{*, \epsilon}}}^{\varphi}={\overline{\partial_{j} \mathbf{U}_{*, \epsilon}}}^{\varphi} \longrightarrow{\overline{\partial_{j} \mathbf{U}_{*}}}^{\varphi}=\partial_{j}{\overline{\mathbf{U}_{*}}}^{\varphi} \quad \text { in } C\left([0, l] ; H^{-1}(S)\right), \\
{\overline{\mathbf{U}_{*, \epsilon}}}^{\varphi} \longrightarrow{\overline{\mathbf{U}_{*}}}^{\varphi}=\mathbf{U}_{*, \varphi}, \quad \text { in } L^{2}(\Omega) .
\end{gathered}
$$

\section{The main result}

In this section, we pass from the three-dimensional model (4.13)-(4.14) to the asymptotic model and our main result is stated and proved.

Let us mention for the reader's convenience that we have proved in Corollary 5.3 that

$$
\begin{gathered}
\mathbf{U}_{\epsilon_{n}} \stackrel{*}{\longrightarrow} \mathbf{U} \quad \text { in } L^{\infty}\left(0, T ; V(\Omega)^{3}\right), \quad \partial_{t} \mathbf{U}_{\epsilon} \stackrel{*}{\longrightarrow} \partial_{t} \mathbf{U} \quad \text { in } L^{\infty}\left(0, T ; L^{2}(\Omega)^{3}\right), \\
\frac{1}{\epsilon_{n}} \omega^{\epsilon_{n}}\left(\mathbf{U}_{\epsilon_{n}}\right) \stackrel{*}{\longrightarrow} \zeta \quad \text { in } L^{\infty}\left(0, T ; L^{2}(\Omega)^{9}\right),
\end{gathered}
$$

for $\epsilon_{n} \rightarrow 0$, where $\mathbf{U} \in L^{\infty}\left(0, T ; H_{0}^{1}(0, l)^{3}\right)$ according to Proposition 6.1.

Now, we mention without proofs two propositions and one corollary, because the proofs can be obtained similarly as in [14]. 
Proposition 7.1. Let the tensor $\zeta$ be the limit determined by (7.2). Then it satisfies the equation

$$
\int_{\Omega} A_{0}^{i j k l} \zeta_{k l}(t) \theta_{i j}^{0}(\mathbf{V}) d x=0
$$

for all $\mathbf{V} \in L^{2}\left(0, l ; H^{1}(S)^{3}\right)$ and for a.a. $t \in(0, T)$, where the tensor $\theta^{0}(\mathbf{V})$ is defined by

$$
\theta^{0}(\mathbf{V})=\left(\begin{array}{ccc}
0 & \frac{\left(\partial_{2} \mathbf{V}, \mathbf{t}\right)}{2} & \frac{\left(\partial_{3} \mathbf{V}, \mathbf{t}\right)}{2} \\
\frac{\left(\partial_{2} \mathbf{V}, \mathbf{t}\right)}{2} & \left(\partial_{2} \mathbf{V}, \mathbf{n}\right) & \frac{\left(\partial_{2} \mathbf{V}, \mathbf{b}\right)+\left(\partial_{3} \mathbf{V}, \mathbf{n}\right)}{2} \\
\frac{\left(\partial_{3} \mathbf{V}, \mathbf{t}\right)}{2} & \frac{\left(\partial_{2} \mathbf{V}, \mathbf{b}\right)+\left(\partial_{3} \mathbf{V}, \mathbf{n}\right)}{2} & \left(\partial_{3} \mathbf{V}, \mathbf{b}\right)
\end{array}\right) .
$$

We introduce the following notation:

$$
\zeta_{22}^{H}=\zeta_{22}+\frac{1}{2} \frac{\lambda}{\lambda+\mu} \zeta_{11}, \quad \zeta_{33}^{H}=\zeta_{33}+\frac{1}{2} \frac{\lambda}{\lambda+\mu} \zeta_{11}, \quad \zeta_{23}^{H}=\zeta_{23} .
$$

Corollary 7.2. We have

$$
\begin{aligned}
\int_{S} \zeta_{12} d x_{2} d x_{3} & =\int_{S} \zeta_{13} d x_{2} d x_{3}=\int_{S} \zeta_{12} x_{2} d x_{2} d x_{3} \\
& =\int_{S} \zeta_{13} x_{3} d x_{2} d x_{3}=\int_{S}\left[\zeta_{12} x_{3}+\zeta_{13} x_{2}\right] d x_{2} d x_{3}=0, \\
\int_{S} \zeta_{23}^{H} d x_{2} d x_{3} & =\int_{S} \zeta_{23}^{H} x_{2} d x_{2} d x_{3}=\int_{S} \zeta_{23}^{H} x_{3} d x_{2} d x_{3}=0, \\
\int_{S}\left(\zeta_{22}^{H}+\zeta_{33}^{H}\right) d x_{2} d x_{3} & =\int_{S}\left(\zeta_{22}^{H}+\zeta_{33}^{H}\right) x_{2} d x_{2} d x_{3} \\
& =\int_{S}\left(\zeta_{22}^{H}+\zeta_{33}^{H}\right) x_{3} d x_{2} d x_{3}=0,
\end{aligned}
$$

a.e. in $(0, l) \times(0, T)$.

If we define the vector $\boldsymbol{\eta} \in L^{\infty}\left(0, T ; L^{2}(\Omega)^{2}\right)$ by $\boldsymbol{\eta}=\left\lfloor\zeta_{12}, \zeta_{13}\right\rfloor$, the equations (7.3), after putting $\mathbf{V}=\varphi \mathbf{t}, \varphi \in L^{2}\left(0, l ; H^{1}(S)\right)$, and (6.5) can be rewritten in the form

$$
\begin{aligned}
& \int_{\Omega}\left(\boldsymbol{\eta}(t), \nabla_{23} \varphi\right)_{2} d x=0, \quad \forall \varphi \in L^{2}\left(0, l ; H^{1}(S)\right), \\
& \int_{\Omega}\left(\boldsymbol{\eta}(t), \operatorname{rot}_{23} \psi\right)_{2} d x=\int_{\Omega}\left(\mathbf{U}_{*}^{\prime}(t), \mathbf{t}\right) \psi d x, \quad \forall \psi \in H_{0}^{1}(\Omega),
\end{aligned}
$$

for a.a. $t \in(0, T)$, where we have denoted $\nabla_{23} \varphi=\left\lfloor\partial_{2} \varphi, \partial_{3} \varphi\right\rfloor, \operatorname{rot}_{23} \psi=\left\lfloor-\partial_{3} \psi, \partial_{2} \psi\right\rfloor$, and where $(\cdot, \cdot)_{2}$ means the scalar product in the usual two-dimensional Euclidean space $\mathbb{R}^{2}$.

Proposition 7.3. Under the assumptions on the domain S from Section 2, the system (7.9), (7.10) has the unique solution in $L^{\infty}\left(0, T ; L^{2}(\Omega)^{2}\right)$ given by

$$
\eta=\left\lfloor\zeta_{12}, \zeta_{13}\right\rfloor=-\frac{1}{2}\left(\partial_{1} \mathbf{U}_{*}, \mathbf{t}\right)\left\lfloor\partial_{2} p-x_{3}, \partial_{3} p+x_{2}\right\rfloor
$$


where the function $p \in H^{1}(S)$ is the unique solution to the Neumann problem

$$
\int_{S}\left[\left(\partial_{2} p-x_{3}\right) \partial_{2} r+\left(\partial_{3} p+x_{2}\right) \partial_{3} r\right] d x_{2} d x_{3}=0, \quad \int_{S} p d x_{2} d x_{3}=0
$$

for all $r \in H^{1}(S)$.

Now, we derive the asymptotic model. First we introduce some constants:

$$
\begin{gathered}
I_{1}=\int_{S} x_{2}^{2} d x_{2} d x_{3}, \quad I_{2}=\int_{S} x_{3}^{2} d x_{2} d x_{3}, \\
E=\mu \frac{3 \lambda+2 \mu}{\lambda+\mu}, \quad K=\int_{S}\left[\left(\partial_{2} p-x_{3}\right)^{2}+\left(\partial_{3} p+x_{2}\right)^{2}\right] d x_{2} d x_{3},
\end{gathered}
$$

where $p \in H^{1}(S)$ is the unique solution to the Neumann problem (7.12).

Lemma 7.4. Let $\left\{\mathbf{U}_{\epsilon_{n}}\right\}_{n=1}^{\infty}, \epsilon_{n} \rightarrow 0$, be a subsequence of the weak solutions to the problem (4.13)-(4.14) satisfying (5.1), (7.1)-(7.2). Then the limit $\lfloor\mathbf{U}, \phi\rfloor \in L^{\infty}\left(0, T ; \mathfrak{V}_{0}^{\mathbf{t}, \mathbf{n}, \mathbf{b}}(0, l)\right) o b$ tained in Proposition 6.1 generates the function $\mathbf{U}_{*}$, which satisfies the equation

$$
\begin{aligned}
& -\int_{0}^{l} \check{\rho}\left({\overline{\partial_{t} \mathbf{U}}}^{\dot{\varphi}}, \mathbf{V}\right) d x_{1}+\int_{0}^{l} E\left[I_{1}\left({\overline{\partial_{1} \mathbf{U}_{*}}}^{\varphi}, \mathbf{b}\right)\left(\mathbf{V}_{*}^{\prime}, \mathbf{b}\right)+I_{2}\left({\overline{\partial_{1} \mathbf{U}_{*}}}^{\varphi}, \mathbf{n}\right)\left(\mathbf{V}_{*}^{\prime}, \mathbf{n}\right)\right] d x_{1} \\
& \quad+\int_{0}^{l} \mu K\left({\overline{\partial_{1} \mathbf{U}_{*}}}^{\varphi}, \mathbf{t}\right)\left(\mathbf{V}_{*}^{\prime}, \mathbf{t}\right) d x_{1}=\int_{0}^{l}\left({\overline{\check{F}_{\mathrm{F}+\mathbf{G}}}}^{\varphi}, \mathbf{V}\right) d x_{1}
\end{aligned}
$$

for all functions $\mathbf{V}_{*} \in H_{0}^{1}(0, l)^{3}$ generated by an arbitrary couple $\lfloor\mathbf{V}, \psi\rfloor \in \mathscr{V}_{0}^{\mathbf{t}, \mathbf{n}, \mathbf{b}}(0, l)$ (see (2.9)) and for all functions $\varphi \in C_{0}^{\infty}(0, T)$, where $\check{\mathbf{F}}_{\mathbf{F}+\mathbf{G}}\left(t, x_{1}\right)=\int_{S} \mathbf{F}\left(t, x_{1}\right) d x_{2} d x_{3}+\int_{\partial S} \mathbf{G}(t$, $\left.x_{1}\right) d S$ and $\check{\rho}\left(x_{1}\right)=\int_{S} \rho\left(x_{1}\right) d x_{2} d x_{3},\left(x_{1}, t\right) \in(0, l) \times(0, T)$.

Proof. Let $\lfloor\mathbf{V}, \psi\rfloor$ be an arbitrary couple of functions from the space $\mathscr{V}_{0}^{\mathbf{t}, \mathbf{n}, \mathbf{b}}(0, l)$ and the couples $\left\lfloor\mathbf{V}_{\epsilon}, \psi_{\epsilon}\right\rfloor \in \mathscr{V}_{0}^{\mathbf{t}_{\epsilon}, \mathbf{n}_{\epsilon}, \mathbf{b}_{\epsilon}}(0, l)$ its smooth approximations given by Proposition 3.1. We define the functions $\mathbf{W}_{\epsilon} \in C^{\infty}(\bar{\Omega})^{3}$ and $\hat{\mathbf{V}}_{\epsilon} \in C^{\infty}(\bar{\Omega})^{3} \cap V(\Omega)^{3}$ by

$$
\begin{gathered}
\mathbf{W}_{\epsilon}\left(x_{1}, x_{2}, x_{3}\right)=-\left(\left(\mathbf{V}_{\epsilon}^{\prime}\left(x_{1}\right), \mathbf{n}_{\epsilon}\left(x_{1}\right)\right) x_{2}+\left(\mathbf{V}_{\epsilon}^{\prime}\left(x_{1}\right), \mathbf{b}_{\epsilon}\left(x_{1}\right)\right) x_{3}\right) \mathbf{t}_{\epsilon}\left(x_{1}\right) \\
-x_{3} \psi_{\epsilon}\left(x_{1}\right) \mathbf{n}_{\epsilon}\left(x_{1}\right)+x_{2} \psi_{\epsilon}\left(x_{1}\right) \mathbf{b}_{\epsilon}\left(x_{1}\right) \quad \text { for }\left(x_{1}, x_{2}, x_{3}\right) \in \Omega, \\
\hat{\mathbf{V}}_{\epsilon}=\mathbf{V}_{\epsilon}+\epsilon \mathbf{W}_{\epsilon} .
\end{gathered}
$$

Using (2.5), (2.16), (4.6)-(4.9), we can verify analogously as in the proof of [14, Lemma 8.4] that

$$
\omega^{\epsilon}\left(\hat{\mathbf{V}}_{\epsilon}\right)=\epsilon \Upsilon\left(\mathbf{V}_{*, \epsilon}\right)+B_{\epsilon}
$$


where

$$
\begin{gathered}
\Upsilon_{11}\left(\mathbf{V}_{*, \epsilon}\right)=-\left(\mathbf{V}_{*, \epsilon}^{\prime}, \mathbf{n}_{\epsilon}\right) x_{3}+\left(\mathbf{V}_{*, \epsilon}^{\prime}, \mathbf{b}_{\epsilon}\right) x_{2}, \\
\Upsilon_{12}\left(\mathbf{V}_{*, \epsilon}\right)=\Upsilon_{21}\left(\mathbf{V}_{*, \epsilon}\right)=\frac{x_{3}}{2}\left(\mathbf{V}_{*, \epsilon}^{\prime}, \mathbf{t}_{\epsilon}\right), \\
\Upsilon_{13}\left(\mathbf{V}_{*, \epsilon}\right)=\Upsilon_{31}\left(\mathbf{V}_{*, \epsilon}\right)=-\frac{x_{2}}{2}\left(\mathbf{V}_{*, \epsilon}^{\prime}, \mathbf{t}_{\epsilon}\right), \\
\Upsilon_{i j}\left(\mathbf{V}_{*, \epsilon}\right)=0, \quad i, j=2,3, \\
B_{\epsilon}^{11}=\epsilon^{2}\left(\left(\beta_{\epsilon} x_{2}+\alpha_{\epsilon} x_{3}\right)\left(x_{2}\left(\mathbf{V}_{\epsilon}^{\prime}, \mathbf{n}_{\epsilon}\right)^{\prime}+x_{3}\left(\mathbf{V}_{\epsilon}^{\prime}, \mathbf{b}_{\epsilon}\right)^{\prime}-\beta_{\epsilon} x_{3} \psi_{\epsilon}+\alpha_{\epsilon} x_{2} \psi_{\epsilon}\right)\right. \\
\left.+\gamma_{\epsilon} x_{3}\left(\partial_{1} \mathbf{W}_{\epsilon}, \mathbf{n}_{\epsilon}\right)-\gamma_{\epsilon} x_{2}\left(\partial_{1} \mathbf{W}_{\epsilon}, \mathbf{b}_{\epsilon}\right)\right),
\end{gathered}
$$

$B_{\epsilon}^{i j}=0$ for $i, j \neq 1$. Further, we have from (2.2), (2.4), (2.6) and (3.2)-(3.4) that

$$
\begin{gathered}
\Upsilon_{i j}\left(\mathbf{V}_{*, \epsilon}\right) \longrightarrow \Upsilon_{i j}\left(\mathbf{V}_{*}\right) \quad \text { in } L^{2}(\Omega), i, j=1,2,3, \\
\left\|B_{\epsilon}\right\|_{2}=\left\|B_{\epsilon}^{11}\right\|_{2} \leq C \epsilon^{2(1-r)}, \quad r \in\left(0, \frac{1}{3}\right), \\
\hat{\mathbf{V}}_{\epsilon} \longrightarrow \mathbf{V} \quad \text { in } H^{1}(\Omega)^{3}
\end{gathered}
$$

for $\epsilon \rightarrow 0$. In addition, we can deduce from (2.18), (2.19), and (3.5) that

$$
A_{\epsilon}^{i j k l} \longrightarrow A_{0}^{i j k l}=\lambda \delta^{i j} \delta^{k l}+\mu\left(\delta^{i k} \delta^{j l}+\delta^{i l} \delta^{j k}\right) \quad \text { in } C(\bar{\Omega}) .
$$

From the convergences (3.11), (3.12), (7.1)-(7.2), (7.24)-(7.27), it follows that we can pass from the equation

$$
\begin{array}{r}
-\int_{\Omega} \rho\left(\overline{\partial_{t} \mathbf{U}_{\epsilon}}{ }^{\dot{\varphi}}, \hat{\mathbf{V}}_{\epsilon}\right) d_{\epsilon} d x+\int_{\Omega} A_{\epsilon}^{i j k l} \frac{1}{\epsilon} \omega_{k l}^{\epsilon}\left(\overline{\mathbf{U}}_{\epsilon}^{\varphi}\right) \frac{1}{\epsilon} \omega_{i j}^{\epsilon}\left(\hat{\mathbf{V}}_{\epsilon}\right) d_{\epsilon} d x \\
=\int_{\Omega}\left(\overline{\mathbf{F}}^{\varphi}, \hat{\mathbf{V}}_{\epsilon}\right) d_{\epsilon} d x+\int_{0}^{l} \int_{\partial S}\left(\overline{\mathbf{G}}^{\varphi}, \hat{\mathbf{V}}_{\epsilon}\right) d_{\epsilon} \epsilon \sqrt{v_{j} 0^{i j, \epsilon} v_{j}} d S d x_{1}
\end{array}
$$

to

$$
\begin{array}{r}
-\int_{0}^{l} \grave{\rho}\left({\overline{\partial_{t} \mathbf{U}^{\varphi}}}^{\dot{\varphi}} \mathbf{V}\right) d x_{1}+\int_{\Omega} A_{0}^{i j k l} \bar{\zeta}_{k l}^{\varphi} \Upsilon_{i j}\left(\mathbf{V}_{*}\right) d x \\
=\int_{\Omega}\left(\overline{\mathbf{F}}^{\varphi}, \mathbf{V}\right) d x+\int_{0}^{l} \int_{\partial S}\left(\overline{\mathbf{G}}^{\varphi}, \mathbf{V}\right) d S d x_{1}
\end{array}
$$

for all functions $\lfloor\mathbf{V}, \psi\rfloor \in \mathcal{V}_{0}^{\mathbf{t}, \mathbf{n}, \mathbf{b}}(0, l)$, which generate functions $\mathbf{V}_{*}$ (see (2.9)).

It remains to express the second term in (7.29). Equalities (6.6) and (6.7) enable us to express the function $\zeta_{11}$ in this way

$$
\zeta_{11}=Q_{0}+\left(\partial_{1} \mathbf{U}_{*}, \mathbf{b}\right) x_{2}-\left(\partial_{1} \mathbf{U}_{*}, \mathbf{n}\right) x_{3} \quad \text { in } \Omega \times(0, T) .
$$


444 An asymptotic dynamic model for curved rods

Hence and from (2.1), (7.5)-(7.8), (7.11), (7.27), (7.30), we can conclude that

$$
\begin{aligned}
\int_{\Omega} A_{0}^{i j k l} \bar{\zeta}_{k l}^{\varphi} \Upsilon_{i j}\left(\mathbf{V}_{*}\right) d x=\int_{\Omega}\left[\lambda\left(\bar{\zeta}_{11}^{\varphi}+\bar{\zeta}_{22}^{\varphi}+\bar{\zeta}_{33}^{\varphi}\right)+2 \mu \bar{\zeta}_{11}^{\varphi}\right] \Upsilon_{11}\left(\mathbf{V}_{*}\right) d x \\
+\int_{\Omega}\left[4 \mu\left(\bar{\zeta}_{12}^{\varphi} \Upsilon_{12}\left(\mathbf{V}_{*}\right)+\bar{\zeta}_{13}^{\varphi} \Upsilon_{13}\left(\mathbf{V}_{*}\right)\right)\right] d x \\
=\int_{\Omega}\left[\lambda\left(\bar{\zeta}_{11}^{\varphi}+\bar{\zeta}^{\varphi}+\bar{\zeta}^{\varphi}\right)+2 \mu \bar{\zeta}_{11}^{\varphi}\right]\left[\left(\mathbf{V}_{*}^{\prime}, \mathbf{b}\right) x_{2}-\left(\mathbf{V}_{*}^{\prime}, \mathbf{n}\right) x_{3}\right] d x \\
+2 \mu \int_{\Omega}\left[\bar{\zeta}_{12}^{\varphi}\left(\mathbf{V}_{*}^{\prime}, \mathbf{t}\right) x_{3}-\bar{\zeta}_{13}^{\varphi}\left(\mathbf{V}_{*}^{\prime}, \mathbf{t}\right) x_{2}\right] d x \\
=\int_{\Omega}\left[E \bar{\zeta}_{11}^{\varphi}+\lambda\left(\bar{\zeta}_{22}^{\varphi}+\bar{\zeta}_{33}^{\varphi}\right)\right]\left[\left(\mathbf{V}_{*}^{\prime}, \mathbf{b}\right) x_{2}-\left(\mathbf{V}_{*}^{\prime}, \mathbf{n}\right) x_{3}\right] d x \\
+\int_{\Omega} \mu\left(-\left(\partial_{2} p-x_{3}\right) x_{3}+\left(\partial_{3} p+x_{2}\right) x_{2}\right)\left(\partial_{1} \overline{\mathbf{U}}_{*}^{\varphi}, \mathbf{t}\right)\left(\mathbf{V}_{*}^{\prime}, \mathbf{t}\right) d x \\
=\int_{0}^{l} E\left[I_{1}\left({\overline{\partial_{1} \mathbf{U}_{*}}}^{\varphi}, \mathbf{b}\right)\left(\mathbf{V}_{*}^{\prime}, \mathbf{b}\right)+I_{2}\left(\partial_{1} \overline{\mathbf{U}}_{*}^{\varphi}, \mathbf{n}\right)\left(\mathbf{V}_{*}^{\prime}, \mathbf{n}\right)\right] d x_{1} \\
+\int_{0}^{l} \mu K\left({\overline{\partial_{1} \mathbf{U}_{*}}}^{\varphi}, \mathbf{t}\right)\left(\mathbf{V}_{*}^{\prime}, \mathbf{t}\right) d x_{1} .
\end{aligned}
$$

LEMMA 7.5. It holds $Q_{0}=\zeta_{22}^{H}=\zeta_{23}^{H}=\zeta_{33}^{H}=0$ in $\Omega \times(0, T)$.

Proof. According to Proposition 3.2 and (3.11), there exists a constant $C>0$ independent of $\epsilon$ and $\varphi$ such that

$$
\left\|\frac{1}{\epsilon} \omega^{\epsilon}\left(\overline{\mathbf{U}}_{\epsilon}^{\varphi}\right)-\bar{\zeta}^{\varphi}\right\|_{2}^{2} \leq C \Lambda_{\epsilon, \varphi}
$$

for all $\varphi \in C_{0}^{\infty}(0, T)$, where

$$
\Lambda_{\epsilon, \varphi}=\int_{\Omega} A_{\epsilon}^{i j k l}\left(\frac{1}{\epsilon} \omega_{k l}^{\epsilon}\left(\overline{\mathbf{U}}_{\epsilon}^{\varphi}\right)-\bar{\zeta}_{k l}^{\varphi}\right)\left(\frac{1}{\epsilon} \omega_{i j}^{\epsilon}\left(\overline{\mathbf{U}}_{\epsilon}^{\varphi}\right)-\bar{\zeta}_{i j}^{\varphi}\right) d_{\epsilon} d x
$$

Convergences (7.1)-(7.2) and (4.13) imply that

$$
\begin{aligned}
& \Lambda_{\varphi}=\lim _{\epsilon \rightarrow 0} \Lambda_{\epsilon, \varphi} \\
& =\lim _{\epsilon \rightarrow 0}\left[\int_{\Omega}\left(\overline{\mathbf{F}}^{\varphi}, \overline{\mathbf{U}}_{\epsilon}^{\varphi}\right) d_{\epsilon} d x+\int_{0}^{l} \int_{\partial S}\left(\overline{\mathbf{G}}^{\varphi}, \overline{\mathbf{U}}_{\epsilon}^{\varphi}\right) d_{\epsilon} \epsilon \sqrt{\nu_{i} o^{i j, \epsilon} \nu_{j}} d S d x_{1}\right. \\
& +\int_{\Omega} A_{\epsilon}^{i j k l}\left(\left(\bar{\zeta}_{k l}^{\varphi}-\frac{1}{\epsilon} \omega_{k l}^{\epsilon}\left({\overline{\mathbf{U}_{\epsilon}}}^{\varphi}\right)\right) \bar{\zeta}_{i j}^{\varphi}-\bar{\zeta}_{k l}^{\varphi} \frac{1}{\epsilon} \omega_{i j}^{\epsilon}\left({\overline{\mathbf{U}_{\epsilon}}}^{\varphi}\right)\right) d_{\epsilon} d x \\
& \left.+\int_{\Omega} \rho\left({\overline{\partial_{t} \mathbf{U}_{\epsilon}}}^{\dot{\varphi}}, \overline{\mathbf{U}}_{\epsilon}^{\varphi}\right) d_{\epsilon} d x\right] \\
& =\int_{0}^{l}\left(\overline{\mathbf{F}}_{\mathbf{F}+\mathbf{G}}^{\varphi}, \overline{\mathbf{U}}^{\varphi}\right) d x_{1}-\int_{\Omega} A_{0}^{i j k l} \bar{\zeta}_{k l}^{\varphi}{\overline{\zeta_{i j}}}^{\varphi} d x+\int_{0}^{l} \check{\rho}\left({\overline{\partial_{t} \mathbf{U}}}^{\dot{\varphi}}, \overline{\mathbf{U}}^{\varphi}\right) d x_{1} .
\end{aligned}
$$


Using (7.5), (7.11), (7.15), (7.30), we get analogously as in the proof of [14, Lemma 8.5] that

$$
\begin{aligned}
& \int_{\Omega} A_{0}^{i j k l} \bar{\zeta}_{k l}^{\varphi} \bar{\zeta}_{i j}^{\varphi} d x \\
&=\int_{\Omega}\left[E\left({\overline{Q_{0}}}^{\varphi}+\left({\overline{\partial_{1} \mathbf{U}_{*}}}^{\varphi}, \mathbf{b}\right) x_{2}-\left({\overline{\partial_{1} \mathbf{U}_{*}}}^{\varphi}, \mathbf{n}\right) x_{3}\right)^{2}\right. \\
& \quad+4 \mu\left(-\frac{1}{2}\left({\overline{\partial_{1} \mathbf{U}_{*}}}^{\varphi}, \mathbf{t}\right)\left(\partial_{2} p-x_{3}\right)\right)^{2} \\
& \quad+4 \mu\left(-\frac{1}{2}\left({\overline{\partial_{1} \mathbf{U}_{*}}}^{\varphi}, \mathbf{t}\right)\left(\partial_{3} p+x_{2}\right)\right)^{2}+\lambda\left({\overline{\zeta_{22}^{H}}}^{\varphi}+{\overline{\zeta_{33}^{H}}}^{\varphi}\right)^{2} \\
&\left.\quad+2 \mu\left(\left({\overline{\zeta_{22}^{H}}}^{\varphi}\right)^{2}+\left({\overline{\zeta_{33}^{H}}}^{\varphi}\right)^{2}+2\left({\overline{\zeta_{23}^{H}}}^{\varphi}\right)^{2}\right)\right] d x \\
& \quad \int_{0}^{l}\left[\left(\overline{\mathbf{F}}_{\mathbf{F}+\mathbf{G}}^{\varphi}, \overline{\mathbf{U}}^{\varphi}\right)+E|S|\left({\overline{Q_{0}}}^{\varphi}\right)^{2}\right] d x_{1} \\
&+\int_{0}^{l} \check{\rho}\left({\overline{\partial_{t} \mathbf{U}}}^{\dot{\varphi}}, \overline{\mathbf{U}}^{\varphi}\right) d x_{1} \\
& \quad+\int_{\Omega}\left[\lambda\left({\overline{\zeta_{22}^{H}}}^{\varphi}+\bar{\zeta}_{33}^{\varphi}\right)^{2}+2 \mu\left(\left(\bar{\zeta}_{22}^{\varphi}\right)^{2}+\left(\bar{\zeta}_{33}^{\varphi}\right)^{2}+2\left({\overline{\zeta_{23}^{H}}}^{\varphi}\right)^{2}\right)\right] d x .
\end{aligned}
$$

After substitution to (7.34), we obtain

$$
\Lambda_{\varphi}=-\int_{\Omega}\left[E\left({\overline{Q_{0}}}^{\varphi}\right)^{2}+\lambda\left({\overline{\zeta_{22}^{H}}}^{\varphi}+{\overline{\zeta_{33}^{H}}}^{\varphi}\right)^{2}+2 \mu\left(\left({\overline{\zeta_{22}^{H}}}^{\varphi}\right)^{2}+\left({\overline{\zeta_{33}^{H}}}^{\varphi}\right)^{2}+2\left({\overline{\zeta_{23}^{H}}}^{\varphi}\right)^{2}\right)\right] d x
$$

for all $\varphi \in C_{0}^{\infty}(0, T)$. But the sequence $\Lambda_{\epsilon, \varphi}$ for all $\varphi \in C_{0}^{\infty}(0, T)$ consists of non-negative numbers by (7.32) and thus $\Lambda_{\varphi}=0$ for all $\varphi \in C_{0}^{\infty}(0, T)$.

Since we have denoted $\boldsymbol{\eta}=\left\lfloor\zeta_{12}, \zeta_{13}\right\rfloor$, we obtain from Lemma 7.5 that

$$
\begin{gathered}
\zeta_{11} \stackrel{(7.30)}{=}\left(\partial_{1} \mathbf{U}_{*}, \mathbf{b}\right) x_{2}-\left(\partial_{1} \mathbf{U}_{*}, \mathbf{n}\right) x_{3}, \quad \zeta_{12} \stackrel{(7.11)}{=} \zeta_{21}=-\frac{1}{2}\left(\partial_{1} \mathbf{U}_{*}, \mathbf{t}\right)\left(\partial_{2} p-x_{3}\right), \\
\zeta_{13} \stackrel{(7.11)}{=} \zeta_{31}=-\frac{1}{2}\left(\partial_{1} \mathbf{U}_{*}, \mathbf{t}\right)\left(\partial_{3} p+x_{2}\right), \\
\zeta_{22} \stackrel{(7.5)}{=}-\frac{1}{2} \frac{\lambda}{\lambda+\mu}\left(\left(\partial_{1} \mathbf{U}_{*}, \mathbf{b}\right) x_{2}-\left(\partial_{1} \mathbf{U}_{*}, \mathbf{n}\right) x_{3}\right), \quad \zeta_{23}=\zeta_{32}=0, \\
\zeta_{33} \stackrel{(7.5)}{=}-\frac{1}{2} \frac{\lambda}{\lambda+\mu}\left(\left(\partial_{1} \mathbf{U}_{*}, \mathbf{b}\right) x_{2}-\left(\partial_{1} \mathbf{U}_{*}, \mathbf{n}\right) x_{3}\right) .
\end{gathered}
$$

Lemma 7.6. Let the function $\mathbf{U}$ be determined by (7.1) and the functions $\mathbf{Q}_{0}$ and $\mathbf{Q}_{1}$ by (4.12). Then $\left.\mathbf{U}\right|_{t=0}=\mathbf{Q}_{0}$ and $\left.\check{\rho} \partial_{t} \mathbf{U}\right|_{t=0}=\check{\rho} \mathbf{Q}_{1}$ in the sense of the space $C\left([0, T] ; L^{2}(\Omega)^{3}\right)$ or $C\left([0, T] ;\left[\mathscr{V}_{0}^{\mathbf{t}, \mathbf{n}, \mathbf{b}}(0, l)\right]^{\prime}\right)$, respectively.

Proof. The first initial condition follows easily from (4.12), (4.14), and (7.1). Let the function $\hat{\mathbf{V}}_{\epsilon}$ be defined by $(7.17)$ and let $\varphi \in C_{0}^{\infty}(0, T)$ be an arbitrary but fixed function. 
Taking $\varphi \widehat{\mathbf{V}}_{\epsilon}$ as a test function in (4.13), and using (7.18)-(7.23), lead to the equation

$$
\begin{aligned}
-\int_{0}^{T} & \dot{\varphi}(t) \int_{\Omega} \rho\left(\partial_{t} \mathbf{U}_{\epsilon}(t), \hat{\mathbf{V}}_{\epsilon}\right) d_{\epsilon} d x d t+\int_{0}^{T} \varphi(t) \int_{\Omega} A_{\epsilon}^{i j k l} \frac{1}{\epsilon} \omega_{k l}^{\epsilon}\left(\mathbf{U}_{\epsilon}(t)\right) \Upsilon_{i j}\left(\hat{\mathbf{V}}_{\epsilon}\right) d_{\epsilon} d x d t \\
& +\int_{0}^{T} \varphi(t) \int_{\Omega} A_{\epsilon}^{i j k l} \frac{1}{\epsilon} \omega_{k l}^{\epsilon}\left(\mathbf{U}_{\epsilon}(t)\right) \frac{1}{\epsilon} B_{\epsilon}^{i j} d_{\epsilon} d x d t \\
= & \int_{0}^{T} \varphi(t) \int_{\Omega}\left(\mathbf{F}(t), \hat{\mathbf{V}}_{\epsilon}\right) d_{\epsilon} d x d t+\int_{0}^{T} \varphi(t) \int_{0}^{l} \int_{\partial S}\left(\mathbf{G}(t), \hat{\mathbf{V}}_{\epsilon}\right) d_{\epsilon} \epsilon \sqrt{v_{j} o^{i j, \epsilon} v_{j}} d S d x_{1} d t .
\end{aligned}
$$

Equation (7.38) yields that the function $\int_{\Omega} \rho\left(\partial_{t} \mathbf{U}_{\epsilon}(t), \hat{\mathbf{V}}_{\epsilon}\right) d_{\epsilon} d x$ belongs to the space $W^{1, \infty}(0, T)$, which together with (4.14), (5.1), (5.2) enable us to rewrite (7.38) as

$$
\begin{aligned}
\int_{\Omega} \rho\left(\partial_{t} \mathbf{U}_{\epsilon}(t), \hat{\mathbf{V}}_{\epsilon}\right) d_{\epsilon} d x-\int_{\Omega} \rho\left(\mathbf{Q}_{1, \epsilon}, \hat{\mathbf{V}}_{\epsilon}\right) d_{\epsilon} d x \\
=-\int_{0}^{t} \int_{\Omega} A_{\epsilon}^{i j k l} \frac{1}{\epsilon} \omega_{k l}^{\epsilon}\left(\mathbf{U}_{\epsilon}(s)\right) \Upsilon_{i j}\left(\hat{\mathbf{V}}_{\epsilon}\right) d_{\epsilon} d x d s-\int_{0}^{t} \int_{\Omega} A_{\epsilon}^{i j k l} \frac{1}{\epsilon} \omega_{k l}^{\epsilon}\left(\mathbf{U}_{\epsilon}(s)\right) \frac{1}{\epsilon} B_{\epsilon}^{i j} d_{\epsilon} d x d t \\
\quad+\int_{0}^{t} \int_{\Omega}\left(\mathbf{F}(s), \hat{\mathbf{V}}_{\epsilon}\right) d_{\epsilon} d x d s+\int_{0}^{t} \int_{0}^{l} \int_{\partial S}\left(\mathbf{G}(s), \hat{\mathbf{V}}_{\epsilon}\right) d_{\epsilon} \epsilon \sqrt{v_{j} o^{i j, \epsilon} v_{j}} d S d x_{1} d s .
\end{aligned}
$$

We leave to the reader the proof that the right-hand side of $(7.39)$ is convergent in $C([0$, $T]$ ). Hence, from (7.26) and from the second convergence in (7.1), we get that

$$
\int_{\Omega} \rho\left(\partial_{t} \mathbf{U}_{\epsilon}(t)-\mathbf{Q}_{1, \epsilon}, \hat{\mathbf{V}}_{\epsilon}\right) d_{\epsilon} d x \longrightarrow \int_{0}^{l} \check{\rho}\left(\partial_{t} \mathbf{U}(t)-\mathbf{Q}_{1}, \mathbf{V}\right) d x_{1} \quad \text { in } C([0, T]) .
$$

The rest of the proof is obvious.

We have proved that the asymptotic dynamic model for the curved rod has the form:

$$
\begin{aligned}
-\int_{0}^{T} \dot{\varphi}( & t) \int_{0}^{l} \check{\rho}\left(\partial_{t} \mathbf{U}(t), \mathbf{V}\right) d x_{1} d t \\
& +\int_{0}^{T} \varphi(t) \int_{0}^{l} E\left[I_{1}\left(\partial_{1} \mathbf{U}_{*}(t), \mathbf{b}\right)\left(\mathbf{V}_{*}^{\prime}, \mathbf{b}\right)+I_{2}\left(\partial_{1} \mathbf{U}_{*}(t), \mathbf{n}\right)\left(\mathbf{V}_{*}^{\prime}, \mathbf{n}\right)\right] d x_{1} d t \\
& +\int_{0}^{T} \varphi(t) \int_{0}^{l} \mu K\left(\partial_{1} \mathbf{U}_{*}(t), \mathbf{t}\right)\left(\mathbf{V}_{*}^{\prime}, \mathbf{t}\right) d x_{1} d t \\
= & \int_{0}^{T} \varphi(t) \int_{0}^{l}\left(\check{\mathbf{F}}_{\mathbf{F}+\mathbf{G}}(t), \mathbf{V}\right) d x_{1} d t
\end{aligned}
$$

for all functions $\varphi \in C_{0}^{\infty}([0, T])$ and $\mathbf{V}_{*} \in H_{0}^{1}(0, l)^{3}$ generated by an arbitrary couple $\lfloor\mathbf{V}, \psi\rfloor \in \mathscr{V}_{0}^{\mathbf{t}, \mathbf{n}, \mathbf{b}}(0, l)$. The function $\mathbf{U}$, which together with the function $\phi$ generate the function $\mathbf{U}_{*}$ (see (2.9)), satisfies the initial state

$$
\left.\mathbf{U}\right|_{t=0}=\mathbf{Q}_{0},\left.\quad \check{\rho} \partial_{t} \mathbf{U}\right|_{t=0}=\check{\rho} \mathbf{Q}_{1}
$$

in the sense of the space $C\left([0, T] ; L^{2}(0, l)^{3}\right)$ and $C\left([0, T] ;\left[\mathscr{V}_{0}^{\mathbf{t}, \mathbf{n}, \mathbf{b}}(0, l)\right]^{\prime}\right)$, respectively. 
Now, we decide upon the uniqueness of the solution to (7.41)-(7.42).

LEMma 7.7. There exists the unique solution to (7.41) satisfying (7.42).

Proof. Suppose that there exist two solutions $\left\lfloor\mathbf{U}_{j}, \phi_{j}\right\rfloor \in L^{\infty}\left(0, T ; \mathscr{V}_{0}^{\mathbf{t}, \mathbf{n}, \mathbf{b}}(0, l)\right)$ and $\partial_{t} \mathbf{U}_{j} \in$ $L^{\infty}\left(0, T ; L^{2}(0, l)^{3}\right) \cap C\left([0, T] ;\left[\mathscr{V}_{0}^{\mathbf{t}, \mathbf{n}, \mathbf{b}}(0, l)\right]^{\prime}\right), j=1,2$. Let us denote $\hat{\mathbf{U}}=\mathbf{U}_{1}-\mathbf{U}_{2}$ and $\hat{\phi}=$ $\phi_{1}-\phi_{2}$. Then the couple $[\hat{\mathbf{U}}, \hat{\phi}\rfloor \in L^{\infty}\left(0, T ; \mathscr{V}_{0}^{\mathbf{t}, \mathbf{n}, \mathbf{b}}(0, l)\right), \hat{\mathbf{U}}_{*} \in L^{\infty}\left(0, T ; H_{0}^{1}(0, l)^{3}\right), \partial_{t} \hat{\mathbf{U}} \in$ $L^{\infty}\left(0, T ; L^{2}(0, l)^{3}\right) \cap C\left([0, T] ;\left[\mathscr{V}_{0}^{\mathbf{t}, \mathbf{n}, \mathbf{b}}(0, l)\right]^{\prime}\right)$,

$$
\begin{aligned}
-\int_{0}^{T} \dot{\varphi}(t) \int_{0}^{l} \check{\rho}\left(\partial_{t} \hat{\mathbf{U}}(t), \mathbf{V}\right) d x_{1} d t \\
+\int_{0}^{T} \varphi(t) \int_{0}^{l} E\left[I_{1}\left(\partial_{1} \hat{\mathbf{U}}_{*}(t), \mathbf{b}\right)\left(\mathbf{V}_{*}^{\prime}, \mathbf{b}\right)+I_{2}\left(\partial_{1} \hat{\mathbf{U}}_{*}(t), \mathbf{n}\right)\left(\mathbf{V}_{*}^{\prime}, \mathbf{n}\right)\right] \\
\quad+\mu K\left(\partial_{1} \hat{\mathbf{U}}_{*}(t), \mathbf{t}\right)\left(\mathbf{V}_{*}^{\prime}, \mathbf{t}\right) d x_{1} d t=0
\end{aligned}
$$

and the function $\hat{\mathrm{U}}$ satisfies the initial state

$$
\left.\widehat{\mathbf{U}}\right|_{t=0}=0,\left.\quad \grave{\rho} \partial_{t} \hat{\mathbf{U}}\right|_{t=0}=0
$$

in the sense of the space $C\left([0, T] ; L^{2}(0, l)^{3}\right)$ and $C\left([0, T] ;\left[\mathscr{V}_{0}^{\mathbf{t}, \mathbf{n}, \mathbf{b}}(0, l)\right]^{\prime}\right)$, respectively.

From (7.43), it follows that the term $\int_{0}^{l} \check{\rho}\left(\partial_{t} \hat{\mathbf{U}}(t), \mathbf{V}\right) d x d t \in W^{1, \infty}(0, T)$ for all but fixed $\mathbf{V} \in H_{0}^{1}(0, l)^{3}$ such that the couple $\lfloor\mathbf{V}, \psi\rfloor \in \mathscr{V}_{0}^{\mathbf{t}, \mathbf{n}, \mathbf{b}}(0, l)$. This fact enables us to rewrite (7.43) as

$$
\begin{aligned}
& \int_{0}^{l} \check{\rho}\left(\partial_{t} \hat{\mathbf{U}}(t), \mathbf{V}\right) d x_{1}+\int_{0}^{t} \int_{0}^{l} E\left[I_{1}\left(\partial_{1} \hat{\mathbf{U}}_{*}(s), \mathbf{b}\right)\left(\mathbf{V}_{*}^{\prime}, \mathbf{b}\right)+I_{2}\left(\partial_{1} \hat{\mathbf{U}}_{*}(s), \mathbf{n}\right)\left(\mathbf{V}_{*}^{\prime}, \mathbf{n}\right)\right] \\
& \quad+\mu K\left(\partial_{1} \hat{\mathbf{U}}_{*}(s), \mathbf{t}\right)\left(\mathbf{V}_{*}^{\prime}, \mathbf{t}\right) d x_{1} d s=0
\end{aligned}
$$

for all $t \in[0, T]$. Since $\lfloor\hat{\mathbf{U}}(t), \hat{\phi}(t)\rfloor \in \mathscr{V}_{0}^{\mathbf{t}, \mathbf{n}, \mathbf{b}}(0, l)$ for a.a. $t \in(0, T)$, we can use this couple as a test function in (7.45), and we get that

$$
\begin{aligned}
\int_{0}^{l} \check{\rho}\left(\partial_{t} \hat{\mathbf{U}}(t), \hat{\mathbf{U}}(t)\right) d x_{1} \\
\quad+\int_{0}^{t} \int_{0}^{l} E\left[I_{1}\left(\partial_{1} \hat{\mathbf{U}}_{*}(s), \mathbf{b}\right)\left(\partial_{1} \hat{\mathbf{U}}_{*}(t), \mathbf{b}\right)+I_{2}\left(\partial_{1} \hat{\mathbf{U}}_{*}(s), \mathbf{n}\right)\left(\partial_{1} \hat{\mathbf{U}}_{*}(t), \mathbf{n}\right)\right] \\
\quad+\mu K\left(\partial_{1} \hat{\mathbf{U}}_{*}(s), \mathbf{t}\right)\left(\partial_{1} \hat{\mathbf{U}}_{*}(t), \mathbf{t}\right) d x_{1} d s=0
\end{aligned}
$$

for all $t \in[0, T]$. It is obvious that (7.46) can be rewritten as

$$
\begin{aligned}
\frac{d}{d t} \int_{0}^{l} & \frac{\check{\rho}|\hat{\mathbf{U}}(t)|^{2}}{2} d x_{1}+\frac{d}{d t} \int_{0}^{l} \frac{E I_{1}}{2}\left(\int_{0}^{t}\left(\partial_{1} \hat{\mathbf{U}}_{*}(s), \mathbf{b}\right) d s\right)^{2} d x_{1} \\
& +\frac{d}{d t} \int_{0}^{l} \frac{E I_{2}}{2}\left(\int_{0}^{t}\left(\partial_{1} \hat{\mathbf{U}}_{*}(s), \mathbf{n}\right) d s\right)^{2} d x_{1}+\frac{d}{d t} \int_{0}^{l} \frac{\mu K}{2}\left(\int_{0}^{t}\left(\partial_{1} \hat{\mathbf{U}}_{*}(s), \mathbf{t}\right) d s\right)^{2} d x_{1}=0
\end{aligned}
$$


for all $t \in[0, T]$. From the assumptions on the functions $\hat{\mathbf{U}}$ and $\hat{\mathbf{U}}_{*}$ it follows that the functions $\hat{\mathbf{U}}$ and $\int_{0}^{\cdot} \partial_{1} \hat{\mathbf{U}} \in C\left([0, T] ; L^{2}(0, l)^{3}\right)$, which enables us to integrate (7.47) over the interval $[0, t]$, and we get from (7.44) that

$$
\begin{aligned}
& \int_{0}^{l} \frac{\grave{\rho}|\hat{\mathbf{U}}(t)|^{2}}{2} d x_{1}+\int_{0}^{l} \frac{E I_{1}}{2}\left(\int_{0}^{t}\left(\partial_{1} \hat{\mathbf{U}}_{*}(s), \mathbf{b}\right) d s\right)^{2} d x_{1} \\
& \quad+\int_{0}^{l} \frac{E I_{2}}{2}\left(\int_{0}^{t}\left(\partial_{1} \hat{\mathbf{U}}_{*}(s), \mathbf{n}\right) d s\right)^{2} d x_{1}+\int_{0}^{l} \frac{\mu K}{2}\left(\int_{0}^{t}\left(\partial_{1} \hat{\mathbf{U}}_{*}(s), \mathbf{t}\right) d s\right)^{2} d x_{1}=0
\end{aligned}
$$

for all $t \in[0, T]$. Hence $\widehat{\mathbf{U}} \equiv \mathbf{0}$ as a consequence of (4.10) and non-negativity of all terms in (7.48). Further, (7.48) yields that

$$
\begin{aligned}
& \int_{0}^{t} \partial_{1} \hat{\mathbf{U}}_{*}\left(x_{1}, s\right) d s=\int_{0}^{t}\left(\partial_{1} \hat{\mathbf{U}}_{*}\left(x_{1}, s\right), \mathbf{t}\left(x_{1}\right)\right) \mathbf{t}\left(x_{1}\right) \\
& \quad+\left(\partial_{1} \hat{\mathbf{U}}_{*}\left(x_{1}, s\right), \mathbf{n}\left(x_{1}\right)\right) \mathbf{n}\left(x_{1}\right)+\left(\partial_{1} \hat{\mathbf{U}}_{*}\left(x_{1}, s\right), \mathbf{b}\left(x_{1}\right)\right) \mathbf{b}\left(x_{1}\right) d s=0
\end{aligned}
$$

for all $t \in[0, T]$ and for arbitrary but fixed $x_{1} \in(0, l)$. Then $\partial_{1} \mathbf{U}_{*}\left(t, x_{1}\right)=0$ for a.a $\left(x_{1}, t\right) \in$ $(0, l) \times(0, T)$. Since $\mathbf{U}_{*} \in H_{0}^{1}(0, l)$ then also $\mathbf{U}_{*} \equiv \mathbf{0}$ and $\phi=-\left(\mathbf{U}_{*}, \mathbf{t}\right)=0$, a contradiction.

Thus, it is not necessary to pass to subsequences in (7.1) and (7.2).

\section{Transformation of the limit equation}

In this section, we go back to the original curve $\mathscr{C}$ described by the parametrization $\Phi$. We introduce the following notation: $\tilde{v}: \mathscr{C} \rightarrow \mathbb{R}$, where $\tilde{v}\left(\Phi_{1}\left(x_{1}\right), \Phi_{2}\left(x_{1}\right), \Phi_{3}\left(x_{1}\right)\right)=v\left(x_{1}\right)$ for a.a. $x_{1} \in(0, l)$. Then we can easily see that

$$
v^{\prime}\left(x_{1}\right)=\left[\left(\tilde{\partial}_{i} \tilde{v}\right) \circ \Phi\right] t_{i}
$$

and hence

$$
\left[\frac{d}{d x_{1}}(\tilde{v} \circ \boldsymbol{\Phi})\right] \circ \boldsymbol{\Phi}^{-1}=(\tilde{\mathbf{t}}, \tilde{\nabla}) \tilde{v}=\frac{\partial \tilde{v}}{\partial \tilde{\mathbf{t}}}
$$

Using (8.2) we rewrite the limit model (7.41) as follows

$$
\begin{aligned}
& -\int_{0}^{T} \dot{\varphi}(t) \int_{\mathscr{C}} \tilde{\rho}\left(\partial_{t} \tilde{\mathbf{U}}(t), \tilde{\mathbf{V}}\right) d \mathscr{C} d t \\
& +\int_{0}^{T} \varphi(t) \int_{\mathscr{C}} E\left[I_{1}\left(\frac{\partial \tilde{\mathbf{U}}_{*}(t)}{\partial \tilde{\mathbf{t}}}, \tilde{\mathbf{b}}\right)\left(\frac{\partial \tilde{\mathbf{V}}_{*}}{\partial \tilde{\mathbf{t}}}, \tilde{\mathbf{b}}\right)+I_{2}\left(\frac{\partial \tilde{\mathbf{U}}_{*}(t)}{\partial \tilde{\mathbf{t}}}, \tilde{\mathbf{n}}\right)\left(\frac{\partial \tilde{\mathbf{V}}_{*}}{\partial \tilde{\mathbf{t}}}, \tilde{\mathbf{n}}\right)\right] d \mathscr{C} d t \\
& +\int_{0}^{T} \varphi(t) \int_{\mathscr{C}} \mu K\left(\frac{\partial \tilde{\mathbf{U}}_{*}(t)}{\partial \tilde{\mathbf{t}}}, \tilde{\mathbf{t}}\right)\left(\frac{\partial \tilde{\mathbf{V}}_{*}}{\partial \tilde{\mathbf{t}}}, \tilde{\mathbf{t}}\right) d \mathscr{C} d t \\
& =\int_{0}^{T} \varphi(t) \int_{\mathscr{C}}\left(\widetilde{\mathbf{F}}_{\widetilde{\mathbf{F}}+\widetilde{\mathbf{G}}}(t), \tilde{\mathbf{V}}\right) d \mathscr{C} d t, \quad \forall \tilde{\mathbf{V}} \in W_{0}^{1,2}(C) .
\end{aligned}
$$




\section{Example of the limit model for a curve with a lipschitz parametrization}

We finish our paper with a simple example of the limit model (7.41) and (8.3). Let $\mathscr{C}$ be the Jordan unit speed curve defined by the parametrization:

$$
\Phi= \begin{cases}\left(\frac{1}{\sqrt{2}} x_{1}, \frac{1}{\sqrt{2}} x_{1}, 0\right) & \text { if } x_{1} \in\left[0, \frac{1}{2}\right) \\ \left(\frac{1}{\sqrt{2}} x_{1}, \frac{1}{\sqrt{2}}-\frac{1}{\sqrt{2}} x_{1}, 0\right) & \text { if } x_{1} \in\left(\frac{1}{2}, 1\right] .\end{cases}
$$

The appropriate tangent, normal and binormal vectors have the forms

$$
\begin{aligned}
& \mathbf{t}= \begin{cases}\left(\frac{1}{\sqrt{2}}, \frac{1}{\sqrt{2}}, 0\right) & \text { if } x_{1} \in\left[0, \frac{1}{2}\right), \\
\left(\frac{1}{\sqrt{2}},-\frac{1}{\sqrt{2}}, 0\right) & \text { if } x_{1} \in\left(\frac{1}{2}, 1\right],\end{cases} \\
& \mathbf{n}= \begin{cases}\left(\frac{1}{\sqrt{2}},-\frac{1}{\sqrt{2}}, 0\right) & \text { if } x_{1} \in\left[0, \frac{1}{2}\right), \\
\left(-\frac{1}{\sqrt{2}},-\frac{1}{\sqrt{2}}, 0\right) & \text { if } x_{1} \in\left(\frac{1}{2}, 1\right],\end{cases}
\end{aligned}
$$

If we define the mapping $\overline{\mathbf{P}}_{\epsilon} \circ \mathbf{R}_{\epsilon}$ for the function $\boldsymbol{\Phi}$ instead of its smooth approximations (see (2.12)) we get

$$
\overline{\mathbf{P}}_{\epsilon} \circ \mathbf{R}_{\epsilon}= \begin{cases}\left(\frac{1}{\sqrt{2}} x_{1}+\epsilon x_{2} \frac{1}{\sqrt{2}}, \frac{1}{\sqrt{2}} x_{1}-\epsilon x_{2} \frac{1}{\sqrt{2}}, \epsilon x_{3}\right) & \text { if } x_{1} \in\left[0, \frac{1}{2}\right), \\ \left(\frac{1}{\sqrt{2}} x_{1}-\epsilon x_{2} \frac{1}{\sqrt{2}}, \frac{1}{\sqrt{2}}-\frac{1}{\sqrt{2}} x_{1}-\epsilon x_{2} \frac{1}{\sqrt{2}}, \epsilon x_{3}\right) & \text { if } x_{1} \in\left(\frac{1}{2}, 1\right] .\end{cases}
$$

If we put $x_{3}=0, x_{2}=1$ and $x_{1}=1 / 2-\epsilon$ and $x_{1}=1 / 2+\epsilon$, we can check that the mapping $\overline{\mathbf{P}}_{\epsilon} \circ \mathbf{R}_{\epsilon}$ is not one-to-one.

Since $\Phi_{1}^{\prime}=t_{1}>0$ we can use [14, Proposition 3.2] to construct smooth (Jordan) approximations. Then the limit model (7.41) has the form

$$
\begin{gathered}
-\int_{0}^{T} \dot{\varphi}(t) \int_{0}^{1} \check{\rho}\left(\partial_{t} \mathbf{U}(t), \mathbf{V}\right) d x_{1} d t+\int_{0}^{T} \varphi(t) \int_{0}^{1} E I_{1} \partial_{1} U_{3, *}(t) V_{3, *}^{\prime} d x_{1} d t \\
+\frac{E I_{2}}{2} \int_{0}^{T} \varphi(t)\left[\int_{0}^{1 / 2}\left(\partial_{1} U_{1, *}(t)-\partial_{1} U_{2, *}(t)\right)\left(V_{1, *}^{\prime}-V_{2, *}^{\prime}\right) d x_{1}\right. \\
\left.\quad+\int_{1 / 2}^{1}\left(\partial_{1} U_{1, *}(t)+\partial_{1} U_{2, *}(t)\right)\left(V_{1, *}^{\prime}+V_{2, *}^{\prime}\right) d x_{1}\right] d t \\
+\frac{\mu K}{2} \int_{0}^{T} \varphi(t)\left[\int_{0}^{1 / 2}\left(\partial_{1} U_{1, *}(t)+\partial_{1} U_{2, *}(t)\right)\left(V_{1, *}^{\prime}+V_{2, *}^{\prime}\right) d x_{1}\right. \\
\left.\quad+\int_{1 / 2}^{1}\left(\partial_{1} U_{1, *}(t)-\partial_{1} U_{2, *}(t)\right)\left(V_{1, *}^{\prime}-V_{2, *}^{\prime}\right) d x_{1}\right] d t \\
=\int_{0}^{T} \varphi(t) \int_{0}^{1}\left(\check{\mathbf{F}}_{\mathbf{F}+\mathbf{G}}(t), \mathbf{V}\right) d x_{1} d t .
\end{gathered}
$$


Analogously as in the previous section, we transform the limit model as

$$
\begin{aligned}
& -\int_{0}^{T} \dot{\varphi}(t) \int_{C_{1} \cup C_{2}} \tilde{\rho}\left(\partial_{t} \tilde{\mathbf{U}}(t), \tilde{\mathbf{V}}\right) d C d t \\
& +\int_{0}^{T} \varphi(t) \frac{E I_{1}}{2}\left[\int_{C_{1}}\left(\tilde{\partial}_{1} \tilde{U}_{3, *}(t)+\tilde{\partial}_{2} \widetilde{U}_{3, *}(t)\right)\left(\tilde{\partial}_{1} \tilde{V}_{3, *}+\tilde{\partial}_{2} \tilde{V}_{3, *}\right) d C\right. \\
& \left.+\int_{C_{2}}\left(\tilde{\partial}_{1} \tilde{U}_{3, *}(t)-\tilde{\partial}_{2} \tilde{U}_{3, *}(t)\right)\left(\tilde{\partial}_{1} \tilde{V}_{3, *}-\tilde{\partial}_{2} \tilde{V}_{3, *}\right) d C\right] d t \\
& +\int_{0}^{T} \varphi(t) \frac{E I_{2}}{4}\left[\int_{C_{1}}\left(\tilde{\partial}_{1} \tilde{U}_{1, *}(t)+\tilde{\partial}_{2} \tilde{U}_{1, *}(t)-\tilde{\partial}_{1} \tilde{U}_{2, *}(t)-\tilde{\partial}_{2} \tilde{U}_{2, *}(t)\right)\right. \\
& \cdot\left(\tilde{\partial}_{1} \tilde{V}_{1, *}+\tilde{\partial}_{2} \tilde{V}_{1, *}-\tilde{\partial}_{1} \tilde{V}_{2, *}-\tilde{\partial}_{2} \tilde{V}_{2, *}\right) d C \\
& +\int_{C_{2}}\left(\tilde{\partial}_{1} \tilde{U}_{1, *}(t)-\tilde{\partial}_{2} \tilde{U}_{1, *}(t)+\tilde{\partial}_{1} \tilde{U}_{2, *}(t)-\tilde{\partial}_{2} \tilde{U}_{2, *}(t)\right) \\
& \left.\cdot\left(\tilde{\partial}_{1} \tilde{V}_{1, *}-\tilde{\partial}_{2} \tilde{V}_{1, *}+\tilde{\partial}_{1} \tilde{V}_{2, *}-\tilde{\partial}_{2} \tilde{V}_{2, *}\right) d C\right] d t \\
& +\frac{\mu K}{4} \int_{0}^{T} \varphi(t)\left[\int_{C_{1}}\left(\tilde{\partial}_{1} \tilde{U}_{1, *}(t)+\tilde{\partial}_{2} \tilde{U}_{1, *}(t)+\tilde{\partial}_{1} \tilde{U}_{2, *}(t)+\tilde{\partial}_{2} \tilde{U}_{2, *}(t)\right)\right. \\
& \cdot\left(\tilde{\partial}_{1} \tilde{V}_{1, *}+\tilde{\partial}_{2} \tilde{V}_{1, *}+\tilde{\partial}_{1} \tilde{V}_{2, *}+\tilde{\partial}_{2} \tilde{V}_{2, *}\right) d C \\
& +\int_{C_{2}}\left(\tilde{\partial}_{1} \tilde{U}_{1, *}(t)-\tilde{\partial}_{2} \tilde{U}_{1, *}(t)-\tilde{\partial}_{1} \widetilde{U}_{2, *}(t)+\tilde{\partial}_{2} \widetilde{U}_{2, *}(t)\right) \\
& \left.\cdot\left(\tilde{\partial}_{1} \tilde{V}_{1, *}-\tilde{\partial}_{2} \tilde{V}_{1, *}-\tilde{\partial}_{1} \tilde{V}_{2, *}+\tilde{\partial}_{2} \tilde{V}_{2, *}\right) d C\right] d t \\
& =\int_{0}^{T} \varphi(t) \int_{C_{1} \cup C_{2}}\left(\widetilde{\mathbf{F}}_{\widetilde{\mathbf{F}}+\widetilde{\mathbf{G}}(t)}, \tilde{\mathbf{V}}\right) d C d t
\end{aligned}
$$

where the segment $C_{1}$ is given by the equation $\tilde{y}_{2}=\tilde{y}_{1}, \tilde{y}_{1} \in[0,1 / 2 \sqrt{2}]$, and the segment $C_{2}$ by the equation $\tilde{y}_{2}=1 / \sqrt{2}-\tilde{y}_{1}, \tilde{y}_{1} \in[1 / 2 \sqrt{2}, 1 / \sqrt{2}]$.

\section{Acknowledgments}

This paper has been supported by the DFG Research Center FZT 86 "Mathematics for key technologies: Modeling, Simulation, and Optimization of real world processes" in Berlin and by the Council of Czech Government (MSM 6198959214).

\section{References}

[1] J. A. Álvarez-Dios and J. M. Viaño, Mathematical justification of a one-dimensional model for general elastic shallow arches, Math. Methods Appl. Sci. 21 (1998), no. 4, 281-325.

[2] A. Bermúdez and J. M. Viaño, Une justification des équations de la thermoélasticité des poutres à section variable par des méthodes asymptotiques [A justification of thermoelastic equations for variable-section beams by asymptotic methods], RAIRO Anal. Numér. 18 (1984), no. 4, 347-376.

[3] A. Blouza and H. Le Dret, Existence and uniqueness for the linear Koiter model for shells with little regularity, Quart. Appl. Math. 57 (1999), no. 2, 317-337. 
[4] P. G. Ciarlet, Mathematical Elasticity. Vol. III. Theory of Shells, Studies in Mathematics and Its Applications, vol. 29, North-Holland, Amsterdam, 2000.

[5] R. Dautray and J.-L. Lions, Mathematical Analysis and Numerical Methods for Science and Technology. Vol. 5. Evolution Problems. I, Springer, Berlin, 1992.

[6] L. C. Evans, Partial Differential Equations, Graduate Studies in Mathematics, vol. 19, American Mathematical Society, Rhode Island, 1998.

[7] A. Ignat, J. Sprekels, and D. Tiba, A model of a general elastic curved rod, Math. Methods Appl. Sci. 25 (2002), no. 10, 835-854.

[8] M. Jurak and J. Tambača, Derivation and justification of a curved rod model, Math. Models Methods Appl. Sci. 9 (1999), no. 7, 991-1014.

[9] L Linear curved rod model: general curve, Math. Models Methods Appl. Sci. 11 (2001), no. 7, 1237-1252.

[10] A. Raoult, Construction d'un modèle d'évolution de plaques avec terme d'inertie de rotation [Constructing an evolution model for plates with a rotational inertia term], Ann. Mat. Pura Appl. (4) 139 (1985), no. 1, 361-400.

[11] J. Simon, Compact sets in the space $L^{p}(0, T ; B)$, Ann. Mat. Pura Appl. (4) 146 (1987), no. 1, 65-96.

[12] J. Sprekels and D. Tiba, An approximation method for curved rods, Nonlinear Partial Differential Equations and Their Applications (N. Kenmochi, M. Ôtani, and S. Zheng, eds.), GAKUTO Internat. Ser. Math. Sci. Appl., vol. 20, Gakkōtosho, Tokyo, 2004, pp. 305-314.

[13] J. Tambača, Justification of the dynamic model of curved rods, Asymptot. Anal. 31 (2002), no. 1, $43-68$.

[14] D. Tiba and R. Vodák, A general asymptotic model for Lipschitzian curved rods, Adv. Math. Sci. Appl. 15 (2005), no. 1, 137-198.

[15] _ Un modèle asymptotique général pour les poutres courbes lipschitziennes [A general asymptotic model for Lipschitzian curved rods], to appear in C. R. Math. Acad. Sci. Paris.

[16] L. Trabucho and J. M. Viaño, Mathematical modelling of rods, Handbook of Numerical Analysis, Vol. IV (P. G. Ciarlet and J.-L. Lions, eds.), Handb. Numer. Anal., IV, North-Holland, Amsterdam, 1996, pp. 487-974.

[17] Z. Tutek and I. Aganovic, A justification of the one-dimensional linear model of elastic beam, Math. Methods Appl. Sci. 8 (1986), no. 4, 502-515.

[18] L.-M. Xiao, Asymptotic analysis of dynamic problems for linearly elastic shells-justification of equations for dynamic membrane shells, Asymptot. Anal. 17 (1998), no. 2, 121-134.

Rostislav Vodák: Department of Mathematical Analysis and Applications of Mathematics, Faculty of Science, Palacky University, Tř. Svobody 26, 77200 Olomouc, Czech Republic

E-mail address: vodak@inf.upol.cz 


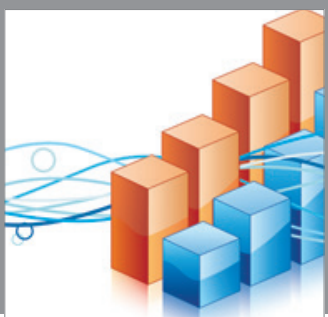

Advances in

Operations Research

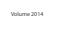

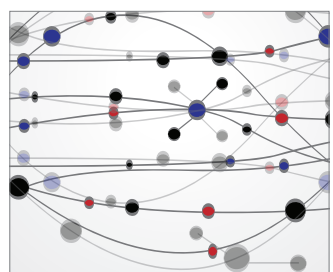

\section{The Scientific} World Journal
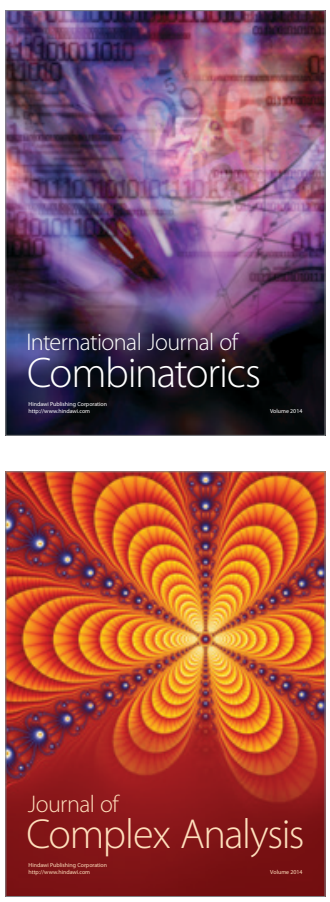

International Journal of

Mathematics and

Mathematical

Sciences
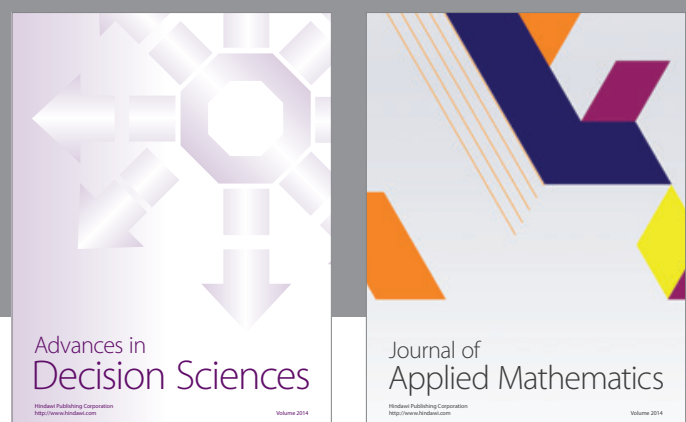

Journal of

Applied Mathematics
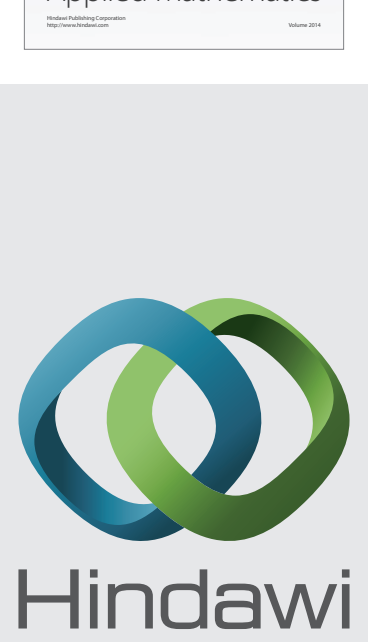

Submit your manuscripts at http://www.hindawi.com
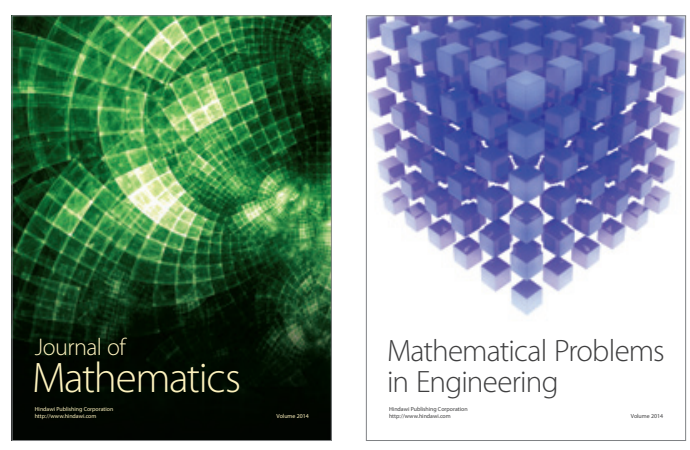

Mathematical Problems in Engineering
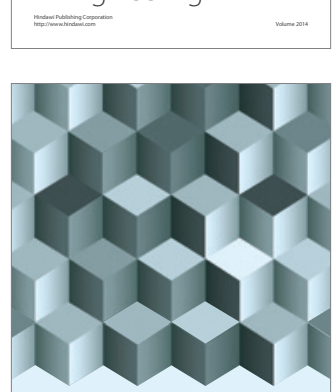

Journal of

Function Spaces
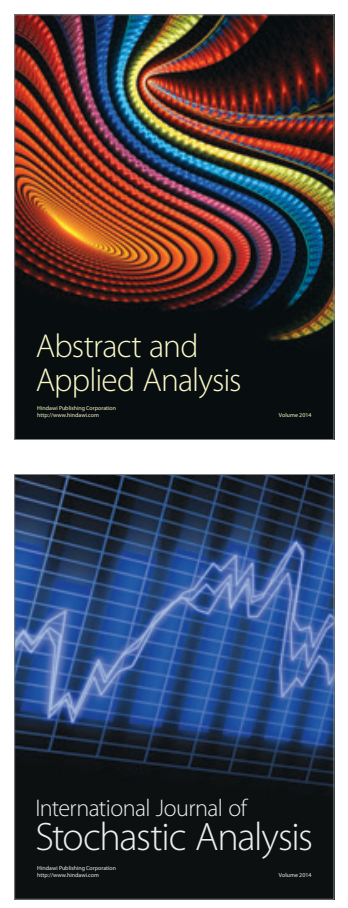

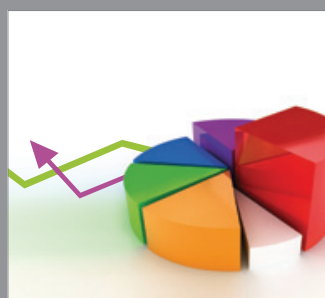

ournal of

Probability and Statistics

Promensencen
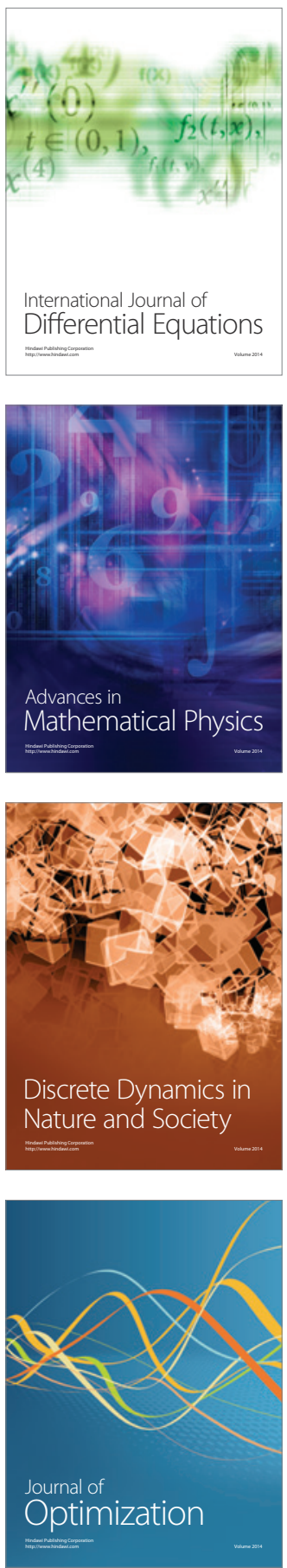